JOURNAL OF

SYMPLECTIC GEOMETRY

Volume 8, Number 3, 323-357, 2010

\title{
LOCAL FLOER HOMOLOGY AND THE ACTION GAP
}

\author{
Viktor L. Ginzburg and Başak Z. Gürel
}

In this paper, we study the behavior of the local Floer homology of an isolated fixed point and the growth of the action gap under iterations. We prove that an isolated fixed point of a diffeomorphism remains isolated for the so-called admissible iterations and that the local Floer homology groups of a Hamiltonian diffeomorphism for such iterations are isomorphic to each other up to a shift of degree. Furthermore, we study the pair-of-pants product in local Floer homology, and characterize a particular class of isolated fixed points (the symplectically degenerate maxima), which plays an important role in the proof of the Conley conjecture. Finally, we apply these results to show that for a quasi-arithmetic sequence of admissible iterations of a Hamiltonian diffeomorphism with isolated fixed points the minimal action gap is bounded from above when the ambient manifold is closed and symplectically aspherical. This theorem is a generalization of the Conley conjecture.

\section{Introduction}

The main objective of this paper is to analyze the behavior of the local Floer homology of an isolated fixed point and of the minimal action gap under iterations of a Hamiltonian diffeomorphism. To be more precise, we prove that an isolated fixed point remains isolated for a certain class of iterations of the diffeomorphism (the so-called admissible iterations) and that the local Floer homology groups for all such iterations persist, i.e., these groups are isomorphic to each other up to a shift of degree. The proofs of these facts rely on an observation of independent interest that for a general diffeomorphism, not necessarily Hamiltonian, an isolated fixed point remains isolated under admissible iterations.

As an application of the persistence of local Floer homology to global Hamiltonian dynamics on closed, symplectically aspherical manifolds, we show that for a quasi-arithmetic sequence of admissible iterations of a 
Hamiltonian diffeomorphism with isolated fixed points the minimal action gap is bounded from above. This theorem can be viewed as a generalization of the Conley conjecture established in $[\mathbf{G i 2}, \mathbf{H i}]$ (see also $[\mathbf{F r H a}, \mathbf{H i}, \mathbf{L e C}]$ ) and asserting that a Hamiltonian diffeomorphism of a closed symplectically aspherical manifold has simple periodic points of arbitrarily large period, see $[\mathbf{C o}]$.

Furthermore, we use the persistence of local Floer homology to study the pair-of-pants product in local Floer homology, and characterize in homological and geometrical terms a particular class of isolated fixed points, the so-called symplectically degenerate maxima, that play an important role in the proof of the Conley conjecture.

1.1. Main results. Let $M$ be a smooth manifold and let $p$ be a fixed point of a diffeomorphism $\varphi: M \rightarrow M$. We call a positive integer $k$ admissible (with respect to $p$ ) if $\lambda^{k} \neq 1$ for all eigenvalues $\lambda \neq 1$ of $d \varphi_{p}: T_{p} M \rightarrow$ $T_{p} M$. In other words, $k$ is admissible if and only if $d \varphi_{p}^{k}$ and $d \varphi_{p}$ have the same generalized eigenvectors with eigenvalue one. For instance, when no eigenvalue $\lambda \neq 1$ is a root of unity, all $k>0$ are admissible. An iteration $k$ is called good with respect to $p$ if the parity of the number of pairs $\left\{\lambda, \lambda^{-1}\right\}$ of negative real eigenvalues, counted with multiplicity, is the same for $d \varphi_{p}^{k}$ and $d \varphi_{p}$. (When $\varphi$ is a symplectomorphism, since the eigenvalue -1 has even multiplicity, the number of pairs is well defined; see, e.g., [SZ].) Otherwise, $k$ is a bad iteration. (These notions are borrowed, with a minor modification convenient in this context, from the construction of contact homology; see, e.g., [Bo].) Furthermore, $k$ is said to be admissible (good) for $\varphi$ if it is admissible (good) with respect to every fixed point of $\varphi$.

Assume now that $M$ is symplectic and $\varphi=\varphi_{H}$ is the time-one map of the Hamiltonian time-dependent flow $\varphi_{H}^{t}$ generated by a one-periodic in time Hamiltonian $H$. Let $\gamma$ be a one-periodic orbit of $H$. Then $k$ is (good) admissible with respect to $\gamma$ if it is (good) admissible for $\varphi_{H}$ with respect to the fixed point $p=\gamma(0)$. In what follows, we denote by $H^{\# k}$ and $\gamma^{k}$ the $k$ th iterations of $H$ and, respectively, $\gamma$. (By definition, the Hamiltonian $H^{\# k}$ generates the time-dependent flow $\left(\varphi_{H}^{t}\right)^{k}$ and $\gamma^{k}(t)=\varphi_{H \# k}^{t}(p)$, where $p=\gamma(0)$ and $t \in[0,1]$. One can think of $\gamma^{k}$ as the $k$-periodic orbit $\gamma(t)$, $t \in[0, k]$, of $H$; see Section 2.1.) When $M$ is symplectically aspherical, the local Floer homology groups $\mathrm{HF}_{*}(H, \gamma)$ are defined similarly to ordinary Floer homology, but taking into account only one-periodic orbits that $\gamma$ splits into under a non-degenerate perturbation of $H$. For instance, when $\gamma$ is non-degenerate, $\operatorname{HF}_{l}(H, \gamma)$ is $\mathbb{Z}_{2}$ if $l$ is equal to the Conley-Zehnder index of $\gamma$ and is zero otherwise. (Here and throughout the rest of the paper, the Floer homology groups are defined using $\mathbb{Z}_{2}$ coefficients.) Let also $\Delta_{H}(\gamma)$ and $A_{H}(\gamma)$ denote the mean index of $\gamma$ and, respectively, the action of $H$ on $\gamma$. We refer the reader to Sections 2.3 and 3 for a detailed discussion of the mean index and local Floer homology, and for further references. Note 
also that in what follows a periodic orbit means, by default, a contractible periodic orbit unless specified otherwise.

The first result of this paper asserts that up to a shift of degree $s_{k}$ the local Floer homology groups $\mathrm{HF}_{*}\left(H^{\# k}, \gamma^{k}\right)$ are isomorphic for all admissible iterations $\varphi_{H}^{k}$ and, moreover, the mean shift $s_{k} / k$ converges to the mean index $\Delta_{H}(\gamma)$. Thus, the general behavior of the local Floer homology groups under iterations is similar to that for non-degenerate periodic orbits.

Theorem 1.1. Let $M$ be a symplectically aspherical manifold and let $\gamma$ be an isolated one-periodic orbit of a Hamiltonian $H: S^{1} \times M \rightarrow \mathbb{R}$. Then $\gamma^{k}$ is also an isolated one-periodic orbit of $H^{\# k}$ for all admissible $k$ and the local Floer homology groups of $H$ and $H^{\# k}$ coincide up to a shift of degree:

$$
\mathrm{HF}_{*+s_{k}}\left(H^{\# k}, \gamma^{k}\right)=\operatorname{HF}_{*}(H, \gamma) \text { for some } s_{k} .
$$

Furthermore, $\lim _{k \rightarrow \infty} s_{k} / k=\Delta_{H}(\gamma)$, and the shift $s_{k}$ is even if $k$ is good, provided that $\mathrm{HF}_{*}(H, \gamma) \neq 0$ and hence the shifts $s_{k}$ are uniquely determined by (1.1). Moreover, when $\Delta_{H}(\gamma)=0$ and $\operatorname{HF}_{n}(H, \gamma) \neq 0$, the orbit $\gamma$ is strongly degenerate (see Section 2.1) and all $s_{k}$ are zero.

Remark 1.1. The assumption that $M$ is symplectically aspherical is not essential in Theorem 1.1. The local Floer homology groups $\operatorname{HF}_{l}(H, \gamma)$ are defined for an arbitrary symplectic manifold $M$, although in this case the orbit $\gamma$ must be equipped with a capping as in the definition of the ordinary Floer homology, [HS]; see, e.g., [GG2] for more details. With this modification, Theorem 1.1, being a local result, holds for any symplectic manifold, as can be easily checked by scrutinizing the proof of Theorem 1.1. The condition that iterations $k$ are admissible is necessary to make sure that $\gamma^{k}$ is isolated; see Proposition 1.1 below. However, it is not clear whether (1.1) fails or holds when the isolation is assumed as is the case in, say, the classical Shub-Sullivan theorem; see $[\mathbf{S S}]$ and the discussion below.

One ingredient of the proof of Theorem 1.1 concerns "persistence of isolation" for fixed points of smooth, not necessarily Hamiltonian, diffeomorphisms and is of independent interest. This is the following result proved in Section 7:

Proposition 1.1. Let $p \in M$ be an isolated fixed point of a $C^{1}$-smooth diffeomorphism $\varphi: M \rightarrow M$. Then $p$ is also an isolated fixed point of $\varphi^{k}$ for every admissible $k$.

Remark 1.2. In the proof of Theorem 1.1, we will also make use of a parametric version of Proposition 1.1. Namely, assume that $p \in M$ is a uniformly isolated fixed point of a family of $C^{1}$-smooth diffeomorphisms $\varphi_{s}: M \rightarrow M$ with $s \in[0,1]$, i.e., $p$ is the only fixed point of $\varphi_{s}$, for all $s$, in some (independent of $s$ ) neighborhood of $p$. Then $p$ is also a uniformly isolated fixed 
point of $\varphi_{s}^{k}$ for every $k$ which is admissible for all $\varphi_{s}$. The proof of this generalization of Proposition 1.1 is given in Section 7 .

One-periodic orbits $\gamma$ with $\Delta_{H}(\gamma)=0$ and $\operatorname{HF}_{n}(H, \gamma) \neq 0$ arise naturally in the proof of the Conley conjecture (see $[\mathbf{G i 2}, \mathbf{H i}]$ ) and are referred to here as symplectically degenerate maxima. Utilizing Theorem 1.1 and the results from $[\mathbf{G i 2}]$, we give homological and geometrical characterizations of symplectically degenerate maxima in Section 5.1. Furthermore, we show that the pair-of-pants product in $\mathrm{HF}_{*}(H, \gamma)$ has strong "vanishing properties" detecting, in particular, symplectically degenerate maxima. Namely, the product is nilpotent if and only if $\gamma$ is not a symplectically degenerate maximum; see Section 5.2.

It is natural to consider Theorem 1.1 in the context of the Shub-Sullivan theorem asserting that whenever $p$ is an isolated fixed point for all iterations $\varphi^{k}$ of a $C^{1}$-smooth map $\varphi$ (which is not required to be a diffeomorphism), the index of $\varphi^{k}$ at $p$ is bounded; see $[\mathbf{S S}]$. In the setting of Theorem 1.1, the index of $\varphi_{H}^{k}$ at $\gamma(0)$ is equal to the Euler characteristic $\sum_{l}(-1)^{l} \operatorname{dim}_{\mathbb{Z}_{2}} \mathrm{HF}_{l}\left(H^{\# k}, \gamma^{k}\right)$. Thus, the absolute value of the index is independent of $k$ and the index is bounded, as long as $k$ is admissible. (However, this consequence of Theorem 1.1 can also be extracted from the proofs of the Shub-Sullivan theorem and of Proposition 1.1, and hence holds in much greater generality, cf. Remark 7.1.) Using Theorem 1.1, it is easy to prove the following literal, Hamiltonian analogue of the Shub-Sullivan theorem:

Corollary 1.1. Let $\gamma$ be a one-periodic orbit of a Hamiltonian $H$ on a symplectically aspherical manifold. Assume that the orbit $\gamma^{k}$ is isolated for all $k>0$. Then $\operatorname{rkHF}_{*}\left(H^{\# k}, \gamma^{k}\right):=\sum_{l} \operatorname{dim}_{\mathbb{Z}_{2}} \operatorname{HF}_{l}\left(H^{\# k}, \gamma^{k}\right)$ is bounded as a function of $k$.

Remark 1.3. In fact, in this corollary and in the Shub-Sullivan theorem, it is sufficient to assume that $\gamma^{k}$ is isolated only for a certain finite collection of $k$. (This is a consequence of Proposition 1.1.) For instance, if no eigenvalue $\lambda \neq 1$ of $d \varphi_{H}$ is a root of unity, it suffices to require $\gamma$ to be isolated.

The analogy with the Shub-Sullivan theorem and with the results of Gromoll and Meyer, [GrMe2], suggests a number of applications of Theorem 1.1 to the existence problem for periodic points of Hamiltonian diffeomorphisms. Namely, for some Hamiltonian diffeomorphisms of non-compact manifolds or symplectomorphisms arising in classical Hamiltonian dynamics, the rank of (filtered) Floer homology appears to grow with the order of iteration, and then the Hamiltonian Shub-Sullivan theorem implies the existence of infinitely many periodic orbits. Here, leaving these applications aside, we focus on just one general result concerning the behavior of the action spectrum of $H^{\# k}$. To state this result, let us recall one more definition. 
A strictly increasing, infinite sequence of positive integers

$$
\nu_{1}<\nu_{2}<\nu_{3}<\cdots
$$

is called quasi-arithmetic if $\nu_{i+1}-\nu_{i}<$ const for all $i$ and some constant independent of $i$. For example, any set containing an infinite arithmetic progression is quasi-arithmetic. Furthermore, it is easy to see that whenever fixed points of $\varphi$ are isolated, the set of (good) admissible iterations is quasiarithmetic. (Indeed, the set of admissible iterations is comprised of integers that are not divisible by the degrees $q_{1}>1, \ldots, q_{r}>1$ of the roots of unity among the eigenvalues $\lambda \neq 1$ of $d \varphi$ at the fixed points of $\varphi$. This set contains the arithmetic progression $m_{k}=1+q_{1} \cdot \ldots \cdot q_{r} \cdot k$. To ensure that the iterations are good, it suffices to add $q_{0}=2$ to the collection of $q_{1}, \ldots, q_{r}$.)

Theorem 1.2. Let $H: S^{1} \times M \rightarrow \mathbb{R}$ be a Hamiltonian on a closed, symplectically aspherical manifold $M$ such that all fixed points of $\varphi_{H}$ are isolated. Then there exist an infinite quasi-arithmetic sequence $\nu_{i}$ of admissible iterations of $\varphi_{H}$, a sequence $y_{i}$ of $\nu_{i}$-periodic orbits of $H$, and a one-periodic orbit $x$ of $H$ such that

- $\left|A_{H^{\# \nu_{i}}}\left(x^{\nu_{i}}\right)-A_{H^{\# \nu_{i}}}\left(y_{i}\right)\right| \leq e$,

- $\left|\Delta_{H \# \nu_{i}}\left(x^{\nu_{i}}\right)-\Delta_{H \# \nu_{i}}\left(y_{i}\right)\right| \leq \delta$,

- $\left|A_{H^{\# \nu_{i}}}\left(x^{\nu_{i}}\right)-A_{H^{\# \nu_{i}}}\left(y_{i}\right)\right|+\left|\Delta_{H^{\# \nu_{i}}}\left(x^{\nu_{i}}\right)-\Delta_{H^{\# \nu_{i}}}\left(y_{i}\right)\right|>0$

for some constants e and $\delta$ independent of $i$. Furthermore, any infinite quasiarithmetic sequence of admissible iterations contains a quasi-arithmetic subsequence $\nu_{i}$ with these properties.

Remark 1.4. Under suitable additional assumptions on $M$ and/or $H$, Theorem 1.2 extends to closed, weakly monotone symplectic manifolds. However, this generalization discussed in [GG2] is far less obvious than the generalization of Theorem 1.1 mentioned in Remark 1.1. Also note that, as simple examples show, the condition that the fixed points of $\varphi_{H}$ are isolated is essential in Theorem 1.2; see, e.g., [Sc3, Example 5.6].

Theorem 1.2 can be readily interpreted as a statement about the behavior of the action and index gaps for the iterations $H^{\# \nu_{i}}$. An action gap of $H$ is the difference $\left|A_{H}\left(\gamma_{1}\right)-A_{H}\left(\gamma_{0}\right)\right|$ for two distinct one-periodic orbits $\gamma_{0}$ and $\gamma_{1}$ of $H$. An index gap is defined in a similar fashion by using the mean index $\Delta_{H}(\gamma)$ in place of $A_{H}(\gamma)$ and an action-index gap is the sum $\Gamma_{H}\left(\gamma_{1}, \gamma_{0}\right):=$ $\left|A_{H}\left(\gamma_{1}\right)-A_{H}\left(\gamma_{0}\right)\right|+\left|\Delta_{H}\left(\gamma_{1}\right)-\Delta_{H}\left(\gamma_{0}\right)\right|$. The connection between the Conley conjecture and the growth of action gaps under iterations can be summarized as the fact that if the Conley conjecture failed to hold, the minimal non-zero action gap would grow linearly with the order of iteration. For instance, the proofs of various versions of the Conley conjecture for Hamiltonians with displaceable support are based on the observation that in this case a certain positive action gap of $H^{\# k}$ remains bounded from above as $k \rightarrow \infty$; see 
[FS, Gü, HZ, Sc3, Vi1]. (For such Hamiltonians, the Conley conjecture asserts the existence of simple periodic points with non-zero action and arbitrarily large period, provided that $\varphi_{H} \neq \mathrm{id}$.) Yet, although Theorem 1.2 does ensure that certain action gaps remain bounded, it does not guarantee that these gaps are non-zero. This difficulty is overcome once action gaps are replaced by action-index gaps, and hence, Theorem 1.2 still implies the Conley conjecture.

Corollary 1.2. [Gi2] Let $\varphi: M \rightarrow M$ be a Hamiltonian diffeomorphism of a closed, symplectically aspherical manifold $M$. Assume that the fixed points of $\varphi$ are isolated. Then $\varphi$ has simple periodic orbits of arbitrarily large period.

Proof. Assume the contrary: $\varphi$ has only finitely many simple periodic orbits. Let $p_{0}=1, p_{1}>1, \ldots, p_{l}>1$ be the periods of these orbits. As above, denote by $q_{1}>1, \ldots, q_{r}>1$ the degrees of the roots of unity among the eigenvalues $\lambda \neq 1$ of $d \varphi$ at the fixed points of $\varphi$. The integers not divisible by $p_{1}, \ldots, p_{l}, q_{1}, \ldots, q_{r}$ are admissible and form a quasi-arithmetic sequence. Pick a sequence of iterations $\nu_{i}$ contained in this set such that $0<\Gamma\left(x^{\nu_{i}}, y_{i}\right)<c:=e+\delta$ as in Theorem 1.2. By our choice of $\nu_{i}$, every $\nu_{i}$-periodic orbit is necessarily the $\nu_{i}$ th iteration of a one-periodic orbit and, in particular, $y_{i}=z_{i}^{\nu_{i}}$ for some one-periodic orbits $z_{i}$. As a consequence, $\Gamma\left(x^{\nu_{i}}, y_{i}\right)=\nu_{i} \Gamma\left(x, z_{i}\right)$ and $\Gamma\left(x, z_{i}\right)>0$. Denote by $\epsilon>0$ the minimal positive action-index gap between one-periodic orbits of $\varphi$. Then $\Gamma\left(x, z_{i}\right) \geq \epsilon$ and $\Gamma\left(x^{\nu_{i}}, y_{i}\right) \geq \nu_{i} \epsilon>c$, when $\nu_{i}$ is large enough. This contradicts Theorem 1.2.

Remark 1.5. Let $\varphi$ be a compactly supported, positive Hamiltonian diffeomorphism of $\mathbb{R}^{2 n}$, i.e., $\varphi=\varphi_{H}$, where $H$ is compactly supported, $H \geq 0$, and $H \not \equiv 0$. Then the number of simple periodic orbits of $\varphi$ with positive action and with period less than or equal to $k$ grows at least linearly with $k$, [Vi1]. Moreover, the same is true for any positive Hamiltonian diffeomorphism $\varphi$ of a wide, geometrically bounded manifold (e.g., a manifold convex at infinity) whenever $\varphi$ has compact displaceable support, [Gü]. To the best of the authors' knowledge, no such growth results have been obtained yet either without the positivity assumption or for diffeomorphisms of closed manifolds.

1.2. Organization of the paper. In Section 2, we set conventions and notation, recall the definition and relevant properties of the mean index, and provide some basic references for the construction of Floer homology. Local Floer homology is discussed in Section 3. Theorem 1.1 is proved in Section 4. The questions of homological and geometrical characterization of symplectically degenerate maxima and of vanishing of the pair-of-pants product in local Floer homology are addressed in Section 5. Theorem 1.2 is 
proved in Section 6. The paper is concluded by a proof of Proposition 1.1, given in Section 7 which is independent of the rest of the paper.

\section{Preliminaries}

In this section, we set notation and conventions used in the paper, recall relevant facts concerning Floer homology and the mean index, and provide necessary references for the definitions and proofs.

2.1. Conventions and notation. Throughout the paper, $(M, \omega)$ denotes a symplectic manifold of dimension $2 n$ or, sometimes, $M$ is just a smooth, $m$-dimensional manifold. When $M$ is symplectic, it is always required to be symplectically aspherical, i.e., $\left.\omega\right|_{\pi_{2}(M)}=0=\left.c_{1}(T M)\right|_{\pi_{2}(M)}$, although in some instances (e.g., Theorem 1.1) this requirement can be relaxed. All maps and functions considered in this paper are assumed to be $C^{\infty}$-smooth and all Hamiltonians $H$ are one-periodic in time, i.e., $H: S^{1} \times M \rightarrow \mathbb{R}$, unless specified otherwise. We set $H_{t}=H(t, \cdot)$ for $t \in S^{1}$. The Hamiltonian vector field $X_{H}$ of $H$ is defined by $i_{X_{H}} \omega=-d H$. The time-dependent Hamiltonian flow of $H$, i.e., the flow of $X_{H}$, is denoted by $\varphi_{H}^{t}$. (By definition, a (time dependent) flow is a family of diffeomorphisms beginning at id.) We refer to the time-one map $\varphi_{H}^{1}=: \varphi_{H}$ as a Hamiltonian diffeomorphism. One- or $k$ periodic orbits of $\varphi_{H}^{t}$ are in one-to-one correspondence with fixed points or $k$ periodic points of $\varphi_{H}$. In this paper, we are only concerned with contractible periodic orbits. Thus, reiterating the convention made in the introduction we emphasize that a periodic orbit is always assumed to be contractible, even if this is not explicitly stated.

Let $\gamma: S^{1} \rightarrow M$ be a contractible loop. The action of $H$ on $\gamma$ is given by

$$
A_{H}(\gamma)=-\int_{z} \omega+\int_{S^{1}} H_{t}(\gamma(t)) d t
$$

where $z: D^{2} \rightarrow M$ is such that $\left.z\right|_{S^{1}}=\gamma$. The least action principle asserts that the critical points of $A_{H}$ on the space of all contractible maps $\gamma: S^{1} \rightarrow$ $M$ are exactly the contractible one-periodic orbits of $\varphi_{H}^{t}$.

The action spectrum $\mathcal{S}(H)$ of $H$ is the set of critical values of $A_{H}$. This is a zero measure, closed set; see, e.g., $[\mathbf{H Z}, \mathbf{S c 3}]$. The index spectrum of $H$ is defined in a similar fashion by using the mean index $\Delta_{H}(\gamma)$ in place of $A_{H}(\gamma)$. (The definition and properties of the mean index are reviewed in Section 2.3.) The index spectrum $\mathcal{S}_{\mathcal{I}}(H)$ is a closed set. However, $\mathcal{S}_{\mathcal{I}}(H)$, in contrast with $\mathcal{S}(H)$, need not have zero measure. The action-index spectrum of $H$ is the collection of pairs $\left(A_{H}(\gamma), \Delta_{H}(\gamma)\right) \in \mathbb{R}^{2}$ for all contractible oneperiodic orbits $\gamma$ of $H$; cf. [CFHW]. This is a closed, zero measure subset of $\mathbb{R}^{2}$. Clearly, a non-zero action (index) gap introduced in Section 1.1 is the distance between two points in $\mathcal{S}(H)$ (respectively, $\mathcal{S}_{\mathcal{I}}(H)$ ). 
Definition 2.1. A fixed point $p$ of $\varphi_{H}$ and the one-periodic orbit $\gamma(t)=$ $\varphi_{H}^{t}(p), t \in[0,1]$, are non-degenerate if the linearized return map $d \varphi_{H}: T_{p} M$ $\rightarrow T_{p} M$ has no eigenvalues equal to one. Following [SZ], we call $p$ and $\gamma$ weakly non-degenerate if at least one of the eigenvalues is different from one. Otherwise, $p$ and $\gamma$ are said to be strongly degenerate.

The Conley-Zehnder index of a non-degenerate periodic orbit is defined in $[\mathbf{S a 2}, \mathbf{S Z}]$. In this paper, the Conley-Zehnder index $\mu_{\mathrm{CZ}}(H, \gamma) \in \mathbb{Z}$ of an orbit $\gamma$ is set to be the negative of that in [Sa2]. In other words, we normalize $\mu_{\mathrm{CZ}}$ so that $\mu_{\mathrm{CZ}}(H, \gamma)=n$ when $\gamma$ is a non-degenerate maximum of an autonomous Hamiltonian $H$ with small Hessian. More generally, when $H$ is autonomous and $\gamma$ is a non-degenerate critical point of $H$ such that the eigenvalues of the Hessian (with respect to a metric compatible with $\omega$ ) are less than $2 \pi$, the Conley-Zehnder index of $\gamma$ is equal to one half of the negative signature of the Hessian. When $H$ is clear from the context, we will use the notation $\mu_{\mathrm{CZ}}(\gamma)$.

Furthermore, recall that $\pi_{1}(\operatorname{Sp}(2 n)) \cong \mathbb{Z}$, where $\operatorname{Sp}(2 n)$ is the group of linear symplectic transformations of $\mathbb{R}^{2 n}=\mathbb{C}^{n}$. We fix this isomorphism by requiring it to be the composition of the isomorphism $\pi_{1}(\operatorname{Sp}(2 n)) \cong \pi_{1}(\mathrm{U}(n))$ induced by the inclusion $\mathrm{U}(n) \hookrightarrow \mathrm{Sp}(2 n)$ with the isomorphism $\pi_{1}(\mathrm{U}(n)) \cong$ $\mathbb{Z}$ induced by det: $\mathrm{U}(n) \rightarrow S^{1}$. The Maslov index of a loop in $\operatorname{Sp}(2 n)$ is the class of this loop in $\pi_{1}(\operatorname{Sp}(2 n)) \cong \mathbb{Z}$. As is well known, these definitions carry over unambiguously to the group of linear symplectic transformations of any finite-dimensional symplectic vector space.

Let $K$ and $H$ be two one-periodic Hamiltonians. The composition $K \# H$ is defined by the formula

$$
(K \# H)_{t}=K_{t}+H_{t} \circ\left(\varphi_{K}^{t}\right)^{-1} .
$$

The flow of $K \# H$ is $\varphi_{K}^{t} \circ \varphi_{H}^{t}$. In general, $K \# H$ is not one-periodic. However, this is the case if, for example, $H_{0} \equiv 0 \equiv H_{1}$. The latter condition can be met by reparametrizing the Hamiltonian as a function of time without changing the time-one map. Thus, in what follows, we will always treat $K \# H$ as a one-periodic Hamiltonian. (Another instance when the composition $K \# H$ of two one-periodic Hamiltonians is automatically one-periodic is when the flow $\varphi_{K}^{t}$ is a loop of Hamiltonian diffeomorphisms, i.e., $\varphi_{K}^{1}=$ id.) We set $H^{\# k}=$ $H \# \ldots \# H$ ( $k$ times). The flow $\varphi_{H \# k}^{t}=\left(\varphi_{H}^{t}\right)^{k}, t \in[0,1]$, is homotopic with fixed end-points to the flow $\varphi_{H}^{t}, t \in[0, k]$.

The $k$ th iteration of a one-periodic orbit $\gamma$ of $H$ will be denoted by $\gamma^{k}$. More specifically, $\gamma^{k}(t)=\varphi_{H \# k}^{t}(p)$, where $p=\gamma(0)$ and $t \in[0,1]$. Clearly, $A_{H \# k}\left(\gamma^{k}\right)=k A_{H}(\gamma)$. Replacing $\varphi_{H \# k}^{t}, t \in[0,1]$, by the homotopic flow $\varphi_{H}^{t}, t \in[0, k]$, we can think of $\gamma^{k}$ as the $k$-periodic orbit $\gamma(t), t \in[0, k]$, of $H$. Hence, there is an action-preserving one-to-one correspondence between one-periodic orbits of $H^{\# k}$ and $k$-periodic orbits of $H$. 
A more detailed treatment of the material discussed in this section can be found, for instance, in $[\mathbf{H Z}]$.

2.2. Floer homology. Recall that when $M$ is closed and symplectically aspherical, the filtered Floer homology of $H: S^{1} \times M \rightarrow \mathbb{R}$ for the interval $(a, b)$, denoted throughout the paper by $\mathrm{HF}_{*}^{(a, b)}(H)$, is defined. We refer the reader to Floer's papers $[\mathbf{F l 1}, \mathbf{F 1 2}, \mathbf{F l 3}, \mathbf{F l 4}]$ or to, e.g., $[\mathbf{B P S}, \mathbf{H Z}$, MS, Sa2, SZ, Sc3] for further references and introductory accounts of the construction of (Hamiltonian) Floer homology. Terminology, conventions, and most of the notation used here are identical to those in $[\mathbf{G i 1}, \mathbf{G i 2}, \mathbf{G u ̈}]$.

Consider a one-periodic Hamiltonian $G$ generating a loop of Hamiltonian diffeomorphisms of $M$. Then, as is well known, all orbits $\gamma(t)=\varphi_{G}^{t}(p)$, where $p \in M$ and $t \in S^{1}$, are contractible loops. (This follows from the proof of the Arnold conjecture.) The action $c=A_{G}(\gamma)$ is independent of $p$ and the Maslov index of the linearization $d\left(\varphi_{G}^{t}\right)_{\gamma(t)}$, with respect to the trivialization of $T M$ along $\gamma$ associated with a disk bounded by $\gamma$, is zero. Furthermore, it is easy to see that for a suitable choice of almost complex structures, the composition with the flow of $G$ induces an isomorphism of Floer complexes of $H$ and $G \# H$ shifting the action filtration by $c$ and preserving the grading; see, e.g., $[\mathbf{G i 2}]$ or $[\mathbf{S c 3}]$ and references therein for more details. This isomorphism sends a one-periodic orbit $\gamma$ of $H$ to the one-periodic orbit $\Phi_{G}(\gamma)(t):=\varphi_{G}^{t}(\gamma(t))$ of $G \# H$. Hence, we obtain an isomorphism of Floer homology:

$$
\mathrm{HF}_{*}^{(a, b)}(H) \stackrel{\cong}{\longrightarrow} \mathrm{HF}_{*}^{(a+c, b+c)}(G \# H) .
$$

As a consequence, the filtered Floer homology of $H$ is determined by $\varphi_{H}$ up to a shift of the action filtration.

2.3. The mean index. Let $\gamma$ be a one-periodic orbit of a Hamiltonian $H$ on $M$. (It suffices to have $H$ defined only on a neighborhood of $\gamma$.) The mean index $\Delta_{H}(\gamma) \in \mathbb{R}$ measures the sum of rotations of the eigenvalues of $d\left(\varphi_{H}^{t}\right)_{\gamma(t)}$ lying on the unit circle. Here $d\left(\varphi_{H}^{t}\right)_{\gamma(t)}$ is interpreted as a path in the group of linear symplectomorphisms by using, as above, the trivialization of $T M$ along $\gamma$, associated with a disk bounded by $\gamma$. Referring the reader to $[\mathbf{S Z}]$ for a precise definition of $\Delta_{H}(\gamma)$ and the proofs of its properties, we just recall here the following facts that are used in this paper.

(MI1) The iteration formula: $\Delta_{H \# k}\left(\gamma^{k}\right)=k \Delta_{H}(\gamma)$.

(MI2) Continuity: Let $\tilde{H}$ be a $C^{2}$-small perturbation of $H$ and let $\tilde{\gamma}$ be a one-periodic orbit of $\tilde{H}$ close to $\gamma$. Then $\left|\Delta_{H}(\gamma)-\Delta_{\tilde{H}}(\tilde{\gamma})\right|$ is small.

(MI3) The mean index formula: Assume that $\gamma$ is non-degenerate. Then, as $k \rightarrow \infty$ through admissible iterations, $\mu_{\mathrm{CZ}}\left(H^{\# k}, \gamma^{k}\right) / k \rightarrow \Delta_{H}(\gamma)$.

(MI4) Relation to the Conley-Zehnder index: Let $\gamma$ split into non-degenerate orbits $\gamma_{1}, \ldots, \gamma_{l}$ under a $C^{2}$-small, non-degenerate perturbation $\tilde{H}$ of $H$. Then $\left|\mu_{\mathrm{CZ}}\left(\tilde{H}, \gamma_{i}\right)-\Delta_{H}(\gamma)\right| \leq n$ for all $i=1, \ldots, l$, where 
$n=\operatorname{dim} M / 2$. Moreover, these inequalities are strict when $\gamma$ is weakly non-degenerate; see [SZ, p. 1357]. In particular, if $\gamma$ is non-degenerate, $\left|\mu_{\mathrm{CZ}}(H, \gamma)-\Delta_{H}(\gamma)\right|<n$.

(MI5) Additivity: Let $\gamma_{1}$ and $\gamma_{2}$ be one-periodic orbits of Hamiltonians $H_{1}$ and $H_{2}$ on manifolds $M_{1}$ and, respectively, $M_{2}$. Then $\Delta_{H_{1}+H_{2}}\left(\left(\gamma_{1}, \gamma_{2}\right)\right)=\Delta_{H_{1}}\left(\gamma_{1}\right)+\Delta_{H_{2}}\left(\gamma_{2}\right)$, where $H_{1}+H_{2}$ is the naturally defined Hamiltonian on $M_{1} \times M_{2}$.

(MI6) Action of global loops: Assume that $G$ generates a loop of Hamiltonian diffeomorphisms of $M$. Then $\Delta_{G \# H}\left(\Phi_{G}(\gamma)\right)=\Delta_{H}(\gamma)$.

(MI7) Action of local loops: Assume that $\gamma(t) \equiv p$ is a constant one-periodic orbit and that $G$ generates a loop of Hamiltonian diffeomorphisms fixing $p$ and defined on a neighborhood of $p$. Then $\Delta_{G \# H}\left(\Phi_{G}(\gamma)\right)=$ $\Delta_{H}(\gamma)+2 \mu$, where $\mu$ is the Maslov index of the loop $d\left(\varphi_{G}^{t}\right)_{p}$.

(MI8) Index of strongly degenerate orbits: Assume that $\gamma$ is strongly degenerate. Then $\Delta_{H}(\gamma) \in 2 \mathbb{Z}$. Moreover, when $\gamma \equiv p$ is a constant orbit and $H$ is defined on a neighborhood of $p$ and generates a loop of Hamiltonian diffeomorphisms, we have $\Delta_{H}(p)=2 \mu$, where $\mu$ is the Maslov index of the loop $d\left(\varphi_{H}^{t}\right)_{p}$.

Remark 2.1. Regarding (MI6) and (MI7), note that, as has been pointed out above, the Maslov index of a global loop, in contrast with the index of a local loop, is automatically zero. This ensures that the correction term $2 \mu$ vanishes in the setting of (MI6).

\section{Local Floer homology}

In this section, we briefly recall the definition and basic properties of local Morse and Floer homology following mainly [Gi2], although these constructions go back to the original work of Floer (see, e.g., $[$ F14, F15]) and have been revisited a number of times since then.

3.1. Local Morse homology. Let $f: M^{m} \rightarrow \mathbb{R}$ be a smooth function on a manifold $M$ and let $p \in M$ be an isolated critical point of $f$. Fix a neighborhood $U$ of $p$ containing no other critical points of $f$ and consider a sufficiently small neighborhood $V \subset U$ of $p$ and a generic perturbation $\tilde{f}$ of $f$ in $U$ so that $\left.\tilde{f}\right|_{V}$ is Morse and sufficiently $C^{1}$-close to $f$. Then, as is easy to see, every anti-gradient trajectory connecting two critical points of $\tilde{f}$ in $U$ is entirely contained in $U$. Moreover, the same is true for broken trajectories. As a consequence, the vector space (over $\mathbb{Z}_{2}$ ) generated by the critical points of $\tilde{f}$ in $U$ is a complex with (Morse) differential defined in the standard way; see, e.g., [Jo, Sc1]. Furthermore, the continuation argument shows that the homology of this complex, denoted here by $\operatorname{HM}_{*}(f, p)$ and referred to as the local Morse homology of $f$ at $p$, is independent of the choice of $\tilde{f}$. This construction is a particular case of the one from, e.g., [F14]. 
Example 3.1. Assume that $p$ is a non-degenerate critical point of $f$ of index $k$. Then $\operatorname{HM}_{l}(f, p)=\mathbb{Z}_{2}$ when $l=k$ and $\operatorname{HM}_{l}(f, p)=0$ otherwise.

Example 3.2. When $p$ is a strict local maximum of $f$, we have $\operatorname{HM}_{m}(f, p)=$ $\mathbb{Z}_{2}$. Indeed, in this case, as is easy to see from the standard Morse theory,

$$
\operatorname{HM}_{m}(f, p)=H_{m}(\{f \geq f(p)-\epsilon\},\{f=f(p)-\epsilon\})=\mathbb{Z}_{2},
$$

where $\epsilon>0$ is assumed to be small and such that $f(p)-\epsilon$ is a regular value of $f$. Furthermore, the converse is also true. In fact, $f$ has (strict) local maximum at $p$ if and only if $\operatorname{HM}_{m}(f, p)=\mathbb{Z}_{2}$; see, e.g., [Gi2].

We will need the following property of local Morse homology, which can be easily established by the standard continuation argument; cf. [Sc1].

- Let $f_{s}, s \in[0,1]$, be a family of smooth functions with uniformly isolated critical point $p$, i.e., $p$ is the only critical point of $f_{s}$, for all $s$, in some neighborhood of $p$. Then $\operatorname{HM}_{*}\left(f_{s}, p\right)$ is constant throughout the family, i.e., $\operatorname{HM}_{*}\left(f_{0}, p\right)=\operatorname{HM}_{*}\left(f_{1}, p\right)$.

Remark 3.1. In this observation, the assumption that $p$ is uniformly isolated is essential and cannot be replaced by the weaker condition that $p$ is just an isolated critical point of $f_{s}$ for all $s$. Example: $f_{s}(x)=s x^{2}+(1-s) x^{3}$ on $\mathbb{R}$ with $p=0$. (The authors are grateful to Doris Hein for this remark.)

3.2. Local Floer homology: the definition and basic properties. Let $\gamma$ be an isolated one-periodic orbit of a Hamiltonian $H: S^{1} \times M \rightarrow \mathbb{R}$. Pick a sufficiently small tubular neighborhood $U$ of $\gamma$ and consider a $C^{2}$ small perturbation $\tilde{H}$ of $H$ supported in $U$ such that all periodic orbits of $\tilde{H}$ entering $U$ are non-degenerate. (Such perturbations exist by Salamon and Zehnder [SZ, Theorem 9.1].) Every (anti-gradient) Floer trajectory $u$ connecting two one-periodic orbits of $\tilde{H}$ lying in $U$ is also contained in $U$, provided that $\|\tilde{H}-H\|_{C^{2}}$ and $\operatorname{supp}(\tilde{H}-H)$ are small enough. (This readily follows from the analysis carried out in, e.g., [FHS, Sa1, Sa2].) Thus, by the compactness and gluing theorems, every broken anti-gradient trajectory connecting two such orbits also lies entirely in $U$. Hence, similarly to the definition of local Morse homology, the vector space (over $\mathbb{Z}_{2}$ ) generated by one-periodic orbits of $\tilde{H}$ in $U$ is a complex with (Floer) differential defined in the standard way. The continuation argument (see, e.g., $[\mathbf{S Z}]$ ) shows that the homology of this complex is independent of the choice of $\tilde{H}$ and of the almost complex structure. We refer to the resulting homology group $\operatorname{HF}_{*}(H, \gamma)$ as the local Floer homology of $H$ at $\gamma$. The definition of local Floer homology and most of its properties discussed below extend with natural modifications to all symplectic manifolds, once the orbit $\gamma$ is equipped with a capping; cf. [GG1, Section 6.3.1].

Homology groups of this type were first considered (in a more general setting) by Floer in $[\mathbf{F l 4}, \mathbf{F l 5}]$; see also [Po, Section 3.3.4]. Local Floer and 
Morse homology groups are analogues of (non-equivariant) critical modules introduced in [GrMe1, GrMe2]; see also, e.g., [Lo] for further references.

Example 3.3. Assume that $\gamma$ is non-degenerate and $\mu_{\mathrm{CZ}}(\gamma)=k$. Then $\operatorname{HF}_{l}(H, \gamma)=\mathbb{Z}_{2}$ when $l=k$ and $\operatorname{HF}_{l}(H, \gamma)=0$ otherwise.

In the rest of this section, we list the basic properties of local Floer homology that are essential for what follows.

(LF1) Let $H^{s}, s \in[0,1]$, be a family of Hamiltonians such that $\gamma$ is a uniformly isolated one-periodic orbit for all $H^{s}$, i.e., $\gamma$ is the only periodic orbit of $H_{s}$, for all $s$, in some open set independent of $s$. Then $\operatorname{HF}_{*}\left(H^{s}, \gamma\right)$ is constant throughout the family: $\operatorname{HF}_{*}\left(H^{0}, \gamma\right)=$ $\mathrm{HF}_{*}\left(H^{1}, \gamma\right)$.

The proof of this fact is a straightforward application of the continuation argument; see, e.g., [Gi2]. As in the case of local Morse homology, the condition that $\gamma$ is uniformly isolated is essential.

Local Floer homology spaces are building blocks for filtered Floer homology. Namely, essentially by definition, we have the following:

(LF2) Assume that $M$ is closed and let $c \in \mathbb{R}$ be such that all one-periodic orbits $\gamma_{i}$ of $H$ with action $c$ are isolated. (As a consequence, there are only finitely many orbits with action close to $c$.) Then, if $\epsilon>0$ is small enough,

$$
\mathrm{HF}_{*}^{(c-\epsilon, c+\epsilon)}(H)=\bigoplus_{i} \operatorname{HF}_{*}\left(H, \gamma_{i}\right) .
$$

In particular, if all one-periodic orbits $\gamma$ of $H$ are isolated and $\operatorname{HF}_{k}(H, \gamma)=0$ for some $k$ and all $\gamma$, we have $\operatorname{HF}_{k}(H)=0$ by the long exact sequence of filtered Floer homology.

The local Floer homology is completely determined by the time-one map generated by $H$ :

(LF3) Let $\varphi_{G}^{t}$ be a loop of Hamiltonian diffeomorphisms of $M$. Then

$$
\mathrm{HF}_{*}\left(G \# H, \Phi_{G}(\gamma)\right)=\operatorname{HF}_{*}(H, \gamma)
$$

for every isolated one-periodic orbit $\gamma$ of $H$.

Hence, we will sometimes use the notation $\operatorname{HF}_{*}(\varphi, p)$ for $\operatorname{HF}_{*}(H, \gamma)$, where $\varphi=\varphi_{H}^{1}$ and $p=\gamma(0)$; cf. Section 2.2.

Furthermore, the Künneth formula holds for local Floer homology:

(LF4) Let $\gamma_{1}$ and $\gamma_{2}$ be one-periodic orbits of Hamiltonians $H_{1}$ and $H_{2}$ on, respectively, symplectic manifolds $M_{1}$ and $M_{2}$. Then $\mathrm{HF}_{*}\left(H_{1}+\right.$ $\left.H_{2},\left(\gamma_{1}, \gamma_{2}\right)\right)=\operatorname{HF}_{*}\left(H_{1}, \gamma_{1}\right) \otimes \operatorname{HF}_{*}\left(H_{2}, \gamma_{2}\right)$, where $H_{1}+H_{2}$ is the naturally defined Hamiltonian on $M_{1} \times M_{2}$.

The proofs of (LF2)-(LF4) are straightforward and omitted here for the sake of brevity. (For instance, the proof of (LF4) is identical to the proof of 
the Künneth formula for Floer homology.) We refer the reader to [Gi2] for some more details.

By definition, the support of $\mathrm{HF}_{*}(H, \gamma)$ is the collection of integers $k$ such that $\operatorname{HF}_{k}(H, \gamma) \neq 0$. Clearly, the group $\operatorname{HF}_{*}(H, \gamma)$ is finitely generated and hence supported in a finite range of degrees. The next observation, providing more precise information on the support of $\mathrm{HF}_{*}(H, \gamma)$, is an immediate consequence of (MI4).

(LF5) The group $\operatorname{HF}_{*}(H, \gamma)$ is supported in the range $\left[\Delta_{H}(\gamma)-n, \Delta_{H}(\gamma)+\right.$ $n$ ]. Moreover, when $\gamma$ is weakly non-degenerate, the support is contained in the open interval $\left(\Delta_{H}(\gamma)-n, \Delta_{H}(\gamma)+n\right)$.

As is clear from the definition of local Floer homology, $H$ need not be a function on the entire manifold $M$ - it is sufficient to consider Hamiltonians defined only on a neighborhood of $\gamma$. For the sake of simplicity, we focus on the particular case, relevant here, where $\gamma(t) \equiv p$ is a constant orbit, and hence $d H_{t}(p)=0$ for all $t \in S^{1}$. Then (LF1), (LF4) and (LF5) still hold, and (LF3) takes the following form:

(LF6) Let $\varphi_{G}^{t}$ be a loop of Hamiltonian diffeomorphisms defined on a neighborhood of $p$ and fixing $p$. Then

$$
\mathrm{HF}_{*+2 \mu}(G \# H, p)=\mathrm{HF}_{*}(H, p),
$$

where $\mu$ is the Maslov index of the loop $t \mapsto d\left(\varphi_{G}^{t}\right)_{p} \in \operatorname{Sp}\left(T_{p} M\right)$.

Note that in (LF3), in contrast with (LF6), we a priori have $\mu=0$; cf. Remark 2.1. Hence, the shift of degree does not occur when $\varphi_{G}^{t}$ is a global loop. In other words, comparing (LF3) and (LF6), we can say that while the group $\mathrm{HF}_{*}(H, \gamma)$ is completely determined by $\varphi_{H}: M \rightarrow M$ and $p=\gamma(0)$, the germ of $\varphi_{H}$ at $p$ determines $\operatorname{HF}_{*}(H, p)$ only up to a shift of degree. The degree depends on the class of $\varphi_{H}^{t}$ in the universal covering of the group of germs of Hamiltonian diffeomorphisms.

3.3. Calculation of local Floer homology via local Morse homology. A fundamental property of Floer homology is that $\operatorname{HF}_{*}(H)$ is equal to the Morse homology of $H$ when $H$ is autonomous and $C^{2}$-small; see [FHS, SZ]. A similar identification holds for local Floer homology. In what follows, we will need a slightly more general version of this fact, where the Hamiltonian is, in a certain sense, "nearly" autonomous.

Lemma 3.1. [Gi2] Let $F$ be a smooth function and let $K$ be a one-periodic Hamiltonian, both defined on a neighborhood of a point $p$. Assume that $p$ is an isolated critical point of $F$ and the following conditions are satisfied:

- The inequalities $\left\|X_{K_{t}}(z)-X_{F}(z)\right\| \leq \epsilon\left\|X_{F}(z)\right\|$ and $\left\|\dot{X}_{K_{t}}(z)\right\| \leq$ $\epsilon\left\|X_{F}(z)\right\|$ hold point-wise for $z$ near $p$ for all $t \in S^{1}$. (The dot stands for the derivative with respect to time and, say, $\|X(z)\|$ denotes the norm of the vector $X(z)$.) 
- The Hessians $d^{2}\left(K_{t}\right)_{p}$ and $d^{2} F_{p}$ and the constant $\epsilon \geq 0$ are sufficiently small. Namely, $\epsilon<1$ and

$$
\epsilon(1-\epsilon)^{-1}+\max _{t}\left\|d^{2}\left(K_{t}\right)_{p}\right\|<2 \pi \text { and } \epsilon(1-\epsilon)^{-1}+\left\|d^{2} F_{p}\right\|<2 \pi .
$$

Then $p$ is an isolated one-periodic orbit of $K$ and $\operatorname{HF}_{*}(K, p)=\operatorname{HM}_{*+n}(F, p)$.

Example 3.4. Assume that $p$ is an isolated critical point of an autonomous Hamiltonian $F$ and $\left\|d^{2} F_{p}\right\|<2 \pi$. Then, by Lemma 3.1 with $K=F$, we have $\mathrm{HF}_{*}(F, p)=\operatorname{HM}_{*+n}(F, p)$. This is essentially the isomorphism between Floer and Morse homology for $C^{2}$-small Hamiltonians; see, e.g., [SZ, Section 9]. Note also that the condition that $p$ is an isolated one-periodic orbit of $K$ implies, in particular, that it is an isolated critical point of $K$ when $K$ is autonomous.

To prove Lemma 3.1, one first shows that $p$ is a uniformly isolated oneperiodic orbit for all Hamiltonians from a linear homotopy connecting $K$ and $F$. Thus, $\operatorname{HF}_{*}(K, p)=\operatorname{HF}_{*}(F, p)$ by (LF1). Furthermore, $F$ is $C^{2}$-small near $p$, and thus, by the standard argument (see, e.g., $[\mathbf{F H S}, \mathbf{S Z}]), \mathrm{HF}_{*}(F, p)=$ $\operatorname{HM}_{*+n}(F, p)$. We refer the reader to $[\mathbf{G i 2}]$ for a detailed argument.

Remark 3.2. The requirement in Lemma 3.1 that $K$ is close to an autonomous Hamiltonian $F$ plays a crucial role in the proof of the Conley conjecture, [Gi2, Hi]. To the best of the authors' knowledge, this requirement is originally introduced in [Hi, Lemma 4].

\section{Persistence of local Floer homology}

The main objective of this section is to prove Theorem 1.1. Since the question is local, we may assume without loss of generality that $M=\mathbb{R}^{2 n}$ and $\gamma \equiv p=$ 0 is a constant one-periodic orbit of a germ of a Hamiltonian $H$. Indeed, it is easy to show that the path $\varphi_{H}^{t}, t \in[0,1]$, is homotopic with fixed end-points to a path $\varphi_{\tilde{H}}^{t}$ such that $\varphi_{\tilde{H}}^{t}(p)=p$ for all $t$; see [Gi2, Sections 2.3 and 5.1]. (The argument goes through for a general, not necessarily symplectically aspherical, manifold.) Then $H$ and $\tilde{H}$ have isomorphic graded local Floer homology groups at $p$, and we can just restrict $\tilde{H}$ to a neighborhood of $p$ and use the Darboux theorem. Note also that $p$ is a critical point of $\tilde{H}_{t}$ for all $t$. From now on, we revert to the notation $H$ for the Hamiltonian generating the flow near $p$ and set $\varphi=\varphi_{H}$. The fact that $\gamma^{k} \equiv p$ is isolated, when $k$ is admissible, follows from Proposition 1.1.

The proof of Theorem 1.1 rests on two building blocks. These are the (nearly obvious) case where the fixed point is non-degenerate and the much less trivial case of a strongly degenerate fixed point. Then the Künneth formula implies that the theorem also holds for a split map, i.e., a Hamiltonian diffeomorphism that can be decomposed as the direct product of non-degenerate and strongly degenerate ones. Finally, the general case is 
established by showing that $\varphi$ can be deformed to a split map in the class of Hamiltonian diffeomorphisms with isolated fixed point at the origin. The "moreover" part of the theorem asserting that $p$ is strongly degenerate and all shifts $s_{k}$ are equal to zero if $\Delta_{H}(p)=0$ and $\operatorname{HF}_{n}(H, p) \neq 0$ is proved in Section 4.3 .

Also note that the fact that $s_{k} / k \rightarrow \Delta_{H}(\gamma)$ is clear once (1.1) has been established. Indeed, pick $l$ such that $\operatorname{HF}_{l}(H, \gamma) \neq 0$. Then $\operatorname{HF}_{l_{k}}\left(H^{\# k}, \gamma^{k}\right) \neq$ 0 , where $l_{k}=l+s_{k}$ by (1.1). Furthermore, $\left|l_{k}-\Delta_{H \# k}\left(\gamma^{k}\right)\right| \leq n$ and $\Delta_{H \# k}\left(\gamma^{k}\right)=k \Delta_{H}(\gamma)$ by (LF5) and the iteration formula (MI1). To summarize, $\left|s_{k}+l-k \Delta_{H}(\gamma)\right| \leq n$. Dividing this inequality by $k$, we see that $s_{k} / k \rightarrow \Delta_{H}(\gamma)$. Moreover, $\left|s_{k} / k-\Delta_{H}(\gamma)\right| \leq(n+l) / k$, where $\left|l-\Delta_{H}(\gamma)\right| \leq n$.

4.1. Particular case 1: $\boldsymbol{p}$ is non-degenerate. In this case, the assertion is obvious. Namely, $p$ is a non-degenerate fixed point of $\varphi^{k}$ for every admissible $k$, and hence the Conley-Zehnder index $\mu_{k}$ of $\varphi^{k}$ at $p$ is defined. Clearly,

$$
\operatorname{HF}_{l}\left(\varphi^{k}, p\right)= \begin{cases}\mathbb{Z}_{2} & \text { if } l=\mu_{k} \\ 0 & \text { otherwise }\end{cases}
$$

and the shifts $s_{k}=\mu_{k}-\mu_{1}$ are even when $k$ is good; see [SZ].

4.2. Generating functions. Before turning to the next particular case - that of a strongly degenerate fixed point — we recall in this section a few well-known facts concerning generating functions, which are utilized in Section 4.3. The material reviewed here is absolutely standard - it goes back to Poincaré - and we refer the reader to [Ar, Appendix 9; We71, We77] for more details.

Let us identify $\mathbb{R}^{2 n}$ with the Lagrangian diagonal $\Delta \subset \mathbb{R}^{2 n} \times \overline{\mathbb{R}}^{2 n}$ via the projection $\pi$ to the first factor, where $\mathbb{R}^{2 n} \times \overline{\mathbb{R}}^{2 n}$ is equipped with the symplectic structure $\omega \oplus-\omega$, and fix a Lagrangian complement $N$ to $\Delta$. Thus, $\mathbb{R}^{2 n} \times \overline{\mathbb{R}}^{2 n}$ can now be treated as $T^{*} \Delta$.

Let $\varphi$ be a Hamiltonian diffeomorphism defined on a neighborhood of the origin $p$ in $\mathbb{R}^{2 n}$ and such that $\|\varphi-\mathrm{id}\|_{C^{1}}$ is sufficiently small. Then the graph $\Gamma$ of $\varphi$ is $C^{1}$-close to $\Delta$, and hence $\Gamma$ can be viewed as the graph in $T^{*} \Delta$ of an exact one-form $d F$ near $p \in \Delta=\mathbb{R}^{2 n}$. The function $F$, normalized by $F(p)=0$ and called the generating function of $\varphi$, has the following properties:

(GF1) $p$ is an isolated critical point of $F$ if and only if $p$ is an isolated fixed point of $\varphi$,

(GF2) $\|F\|_{C^{2}}=O\left(\|\varphi-\mathrm{id}\|_{C^{1}}\right)$ and $\left\|d^{2} F_{p}\right\|=O\left(\left\|d \varphi_{p}-I\right\|\right)$.

The function $F$ depends on the choice of the Lagrangian complement $N$ to $\Delta$. To be specific, we take, as $N$, the linear subspace $N_{0}$ of vectors of the form $((x, 0),(0, y))$ in $\mathbb{R}^{2 n} \times \overline{\mathbb{R}}^{2 n}$, where $x=\left(x_{1}, \ldots, x_{n}\right)$ and $y=\left(y_{1}, \ldots, y_{n}\right)$ 
are the standard canonical coordinates on $\mathbb{R}^{2 n}$, i.e., $\omega=\sum d y_{i} \wedge d x_{i}$. Set $z=(x, y)$ and

$$
\psi(z):=(x \text {-component of } \varphi(z), y) .
$$

Then, as is easy to see, $F$ is determined by the equation

$$
\varphi(z)-z=X_{F}(\psi(z))
$$

where $X_{F}$ is the Hamiltonian vector field of $F$. Note also that $N_{0}$, and hence $F$, are uniquely determined by the decomposition of $\mathbb{R}^{2 n}$ into the direct sum of two Lagrangian subspaces - the subspace spanned by $x$ coordinates and the one spanned by $y$-coordinates. Therefore, fixing two transverse Lagrangian subspaces in $\mathbb{R}^{2 n}$ gives rise to a generating function of $\varphi$.

The only reason that above we assumed $\varphi$ to be $C^{1}$-close to id is to make $N$ independent of $\varphi$, and hence make the construction of $F$ to some extent canonical. This assumption can be dropped once more flexibility in the choice of $N$ is allowed. Namely, as is easy to see, for any germ $\varphi$ there exists a Lagrangian complement $N$ to $\Delta$, transverse to the graph of $\varphi$. Then $\varphi$ is given by a generating function with respect to $N$. Conversely, once $N$ is fixed, every function $F$ is the generating function of some Hamiltonian diffeomorphism, provided that the graph of $d F$ in $\mathbb{R}^{2 n} \times \overline{\mathbb{R}}^{2 n}=\Delta \times N=T^{*} \Delta$ is transverse to the fibers of the projection $\pi: \mathbb{R}^{2 n} \times \overline{\mathbb{R}}^{2 n} \rightarrow \mathbb{R}^{2 n}$.

Remark 4.1. From these observations, we recover the well-known fact, used in Section 4.4, that the germ of any symplectomorphism $\varphi$ near a fixed point is Hamiltonian. Indeed, let $F$ be the generating function of $\varphi$ with respect to some $N$. Set $F_{s}=(1-s) F+s d^{2} F_{p}$ with $s \in[0,1]$. Since $d^{2}\left(F_{s}\right)_{p}=d^{2} F_{p}$, the graph of $d F_{s}$ is transverse to the fibers of $\pi$ for all $s$, and we obtain a family $\varphi_{s}$ of symplectic maps fixing $p$ and connecting $\varphi$ to the linear symplectic map $d \varphi$. As a consequence, the germ $\varphi$ lies in the connected component of the identity, and thus, by the standard, elementary argument, $\varphi$ is Hamiltonian.

4.3. Particular case 2: $\boldsymbol{p}$ is strongly degenerate. Since, by definition, all eigenvalues of $d \varphi_{p}$ are equal to one, every $k>0$ is admissible and good. Furthermore, as is easy to check, by a linear symplectic change of coordinates one can make $d \varphi_{p}$ arbitrarily close to identity; see [Gi2, Section 5.2.1]. Hence, we may assume without loss of generality that the iterations $\varphi^{k}$ for all $k$ in an arbitrarily large, but fixed, range are $C^{1}$-close to id in a sufficiently small neighborhood of $p$. As a consequence, $\varphi^{k}$ is given by a generating function $F_{k}$ with respect to $N_{0}$, which is uniquely determined by the equation

$$
\varphi^{k}(z)-z=X_{F_{k}}\left(\psi_{k}(z)\right), \quad F_{k}(p)=0
$$


where, as above,

$$
\psi_{k}(z):=\left(x \text {-component of } \varphi^{k}(z), y\right) .
$$

Set $F=F_{1}$ and $G_{t}=t F_{k}+(1-t) k F$, where $t \in[0,1]$.

Claim 4.1. The origin $p$ is a uniformly isolated critical point of the family $G_{t}, t \in[0,1]$.

Assuming the claim, let us proceed with the proof. First recall that, starting with $F_{k}$, one can construct near $p$ a one-periodic Hamiltonian $K_{k}^{t}$ with time-one map $\varphi^{k}$, satisfying the hypotheses of Lemma 3.1; see [Gi2, Hi]. Then the local Floer homology of $K_{k}$ at $p$ is equal to the local Morse homology of $F_{k}$ at $p$ up to a shift of degree by $n$ :

$$
\mathrm{HF}_{*}\left(K_{k}, p\right)=\operatorname{HM}_{*+n}\left(F_{k}, p\right) .
$$

The Hamiltonians $H^{\# k}$ and $K_{k}$ generate the same time-one map near $p$. Thus,

$$
\operatorname{HF}_{*}\left(K_{k}, p\right)=\operatorname{HF}_{*+m_{k}}\left(H^{\# k}, p\right),
$$

by (LF6), for some even shift of degree $m_{k}$. From the claim and homotopy invariance of local Morse homology (see (LF1)), we infer that

$$
\operatorname{HM}_{*}\left(F_{k}, p\right)=\operatorname{HM}_{*}(k F, p)=\operatorname{HM}_{*}(F, p) .
$$

Hence,

$$
\mathrm{HF}_{*+m_{k}}\left(H^{\# k}, p\right)=\operatorname{HM}_{*+n}\left(F_{k}, p\right)=\operatorname{HM}_{*+n}(F, p)=\operatorname{HF}_{*+m_{1}}(H, p),
$$

and thus (1.1) holds with $s_{k}=m_{k}-m_{1}$. Since all $m_{k}$ are even, the shifts $s_{k}$ are also even.

Now we are in a position to prove the "moreover" part of the theorem. The fact that $p$ is strongly degenerate if $\Delta_{H}(p)=0$ and $\operatorname{HF}_{n}(H, p) \neq 0$ follows immediately from (MI4) or (LF5). It remains to show that $s_{k}=0$ for all $k$. By (MI7),

$$
m_{k}=\Delta_{H \# k}(p)-\Delta_{K_{k}}(p) .
$$

Here $\Delta_{H \# k}(p)=k \Delta_{H}(p)=0$ and $\left|\Delta_{K_{k}}(p)\right|$ is small since $d\left(\varphi_{K_{k}}^{t}\right)_{p}$ is close to the identity. (To be more precise, for a fixed $k$, the path of linear maps $d\left(\varphi_{K_{k}}^{t}\right)_{p}$ can be made arbitrarily close to the identity by a symplectic conjugation.) We conclude that $m_{k}=0$, since $m_{k}$ is an integer, and hence $s_{k}=0$.

Proof of the claim. First, let us show that

$$
\left\|X_{F_{k}}\left(\psi_{k}(z)\right)-k X_{F}(\psi(z))\right\|=O\left(\|\varphi-\mathrm{id}\|_{C^{1}}\right)\left\|X_{F}(\psi(z))\right\|,
$$


where $\psi=\psi_{1}$ and $\|\cdot\|_{C^{1}}$ stands for the $C^{1}$-norm on a sufficiently small ball centered at the origin and, say, $\|X(z)\|$ denotes the norm of the vector $X(z)$. Then, as a consequence of (4.1), we have

$$
\left\|X_{F_{k}}\left(\psi_{k}(z)\right)\right\| \leq\left(k+O\left(\|\varphi-\mathrm{id}\|_{C^{1}}\right)\right)\left\|X_{F}(\psi(z))\right\| .
$$

To prove (4.1), we argue inductively. When $k=1$, the left-hand side is zero and the assertion is obvious. Assume that (4.1), and hence (4.2), have been established for all iterations of order up to and including $k-1$. Then

$$
\begin{aligned}
X_{F_{k}}\left(\psi_{k}(z)\right) & =\varphi^{k}(z)-z \\
& =\left(\varphi^{k}(z)-\varphi^{k-1}(z)\right)+\cdots+(\varphi(z)-z) \\
& =X_{F}\left(\psi \varphi^{k-1}(z)\right)+\cdots+X_{F}(\psi(z)),
\end{aligned}
$$

and therefore

$$
\begin{aligned}
X_{F_{k}}\left(\psi_{k}(z)\right)-k X_{F}(\psi(z))= & X_{F}\left(\psi \varphi^{k-1}(z)\right)-X_{F}(\psi(z)) \\
& +\ldots \\
& +X_{F}(\psi \varphi(z))-X_{F}(\psi(z)) .
\end{aligned}
$$

Furthermore, for every $l$ in the range from 1 to $k-1$, we have

$$
\begin{aligned}
\left\|X_{F}\left(\psi \varphi^{l}(z)\right)-X_{F}(\psi(z))\right\| & \leq\left\|X_{F}\right\|_{C^{1}} \cdot\left\|\psi \varphi^{l}(z)-\psi(z)\right\| \\
& \leq\left\|X_{F}\right\|_{C^{1}} \cdot\|\psi\|_{C^{1}} \cdot\left\|\varphi^{l}(z)-z\right\| \\
& =\left\|X_{F}\right\|_{C^{1}} \cdot\|\psi\|_{C^{1}} \cdot\left\|X_{F_{l}}\left(\psi_{l}(z)\right)\right\| .
\end{aligned}
$$

It is clear that $\left\|X_{F}\right\|_{C^{1}}=O\left(\|F\|_{C^{2}}\right)=O\left(\|\varphi-\mathrm{id}\|_{C^{1}}\right)$ by $(\mathrm{GF} 2)$ and $\|\psi\|_{C^{1}} \leq$ const. Finally, by the induction hypothesis,

$$
\left\|X_{F_{l}}\left(\psi_{l}(z)\right)\right\| \leq\left(l+O\left(\|\varphi-\mathrm{id}\|_{C^{1}}\right)\right)\left\|X_{F}(\psi(z))\right\| .
$$

As a consequence,

$$
\left\|X_{F}\left(\psi \varphi^{l}(z)\right)-X_{F}(\psi(z))\right\| \leq O\left(\|\varphi-\mathrm{id}\|_{C^{1}}\right)\left\|X_{F}(\psi(z))\right\|
$$

for all $l=1, \ldots, k-1$. Adding up these upper bounds for $l=1, \ldots, k-1$ and using (4.3), we obtain (4.1).

Continuing the proof of the claim, we note that it is sufficient to show that $p$ is a uniformly isolated zero of $X_{G_{t}}$. Clearly, for any vector field $Y_{t}$,

$$
\left\|X_{G_{t}}(z)\right\| \geq\left\|Y_{t}(z)\right\|-\left\|Y_{t}(z)-X_{G_{t}}(z)\right\|
$$


Using the linear structure on $\mathbb{R}^{2 n}$, we set

$$
Y_{t}(z)=t X_{F_{k}}\left(\psi_{k}(z)\right)+(1-t) k \cdot X_{F}(\psi(z))
$$

and bound the first term on the right-hand side of (4.4) from below and the second term from above.

By (4.1) and the definition of $Y_{t}(z)$,

$$
\begin{aligned}
\left\|Y_{t}(z)\right\| & \geq k\left\|X_{F}(\psi(z))\right\|-\left\|X_{F_{k}}\left(\psi_{k}(z)\right)-k X_{F}(\psi(z))\right\| \\
& \geq\left(k-O\left(\|\varphi-\mathrm{id}\|_{C^{1}}\right)\right)\left\|X_{F}(\psi(z))\right\| .
\end{aligned}
$$

Next we show that

$$
\left\|Y_{t}(z)-X_{G_{t}}(z)\right\| \leq O\left(\|\varphi-\mathrm{id}\|_{C^{1}}\right)\left\|X_{F}(\psi(z))\right\| .
$$

To this end, first note that

$$
\begin{aligned}
\left\|X_{F}(z)-X_{F}(\psi(z))\right\| & \leq\left\|X_{F}\right\|_{C^{1}} \cdot\|\psi(z)-z\| \\
& \leq\left\|X_{F}\right\|_{C^{1}} \cdot\|\varphi(z)-z\| \\
& \leq\left\|X_{F}\right\|_{C^{1}} \cdot\left\|X_{F}(\psi(z))\right\| \\
& =O\left(\|\varphi-\mathrm{id}\|_{C^{1}}\right)\left\|X_{F}(\psi(z))\right\|,
\end{aligned}
$$

where the second inequality readily follows from the definition of $\psi$. Replacing $F$ by $F_{k}$, we also obtain that

$$
\left\|X_{F_{k}}(z)-X_{F_{k}}\left(\psi_{k}(z)\right)\right\| \leq\left\|X_{F_{k}}\right\|_{C^{1}} \cdot\left\|X_{F_{k}}\left(\psi_{k}(z)\right)\right\| .
$$

Using (4.2), we have $\left\|X_{F_{k}}\right\|=O\left(\left\|X_{F}\right\|_{C^{1}}\right)=O\left(\|\varphi-\mathrm{id}\|_{C^{1}}\right)$, and hence

$$
\left\|X_{F_{k}}(z)-X_{F_{k}}\left(\psi_{k}(z)\right)\right\| \leq O\left(\|\varphi-\mathrm{id}\|_{C^{1}}\right)\left\|X_{F}(\psi(z))\right\| .
$$

Furthermore,

$$
\begin{aligned}
\left\|Y_{t}(z)-X_{G_{t}}(z)\right\| & \leq t\left\|X_{F_{k}}(z)-X_{F_{k}}\left(\psi_{k}(z)\right)\right\|+(1-t) k\left\|X_{F}(z)-X_{F}(\psi(z))\right\| \\
& \leq\left\|X_{F_{k}}(z)-X_{F_{k}}\left(\psi_{k}(z)\right)\right\|+k\left\|X_{F}(z)-X_{F}(\psi(z))\right\| .
\end{aligned}
$$

Combining this with (4.7) and (4.8), we obtain (4.6). Finally, using the bounds (4.5) and (4.6) and inequality (4.4), we conclude that

$$
\left\|X_{G_{t}}(z)\right\| \geq\left(k-O\left(\|\varphi-\mathrm{id}\|_{C^{1}}\right)\right)\left\|X_{F}(\psi(z))\right\| .
$$

It is immediate to see that $\psi$ is a diffeomorphism on a sufficiently small neighborhood of the origin and $\psi(p)=p$. Hence, $\psi(z)=p$ implies that $z=p$. Furthermore, $p$ is a uniformly isolated zero of $X_{F}$ by (GF1). Thus, $p$ is also a uniformly isolated zero of $X_{G_{t}}$. This completes the proof of the claim. 
4.4. Particular case 3: split diffeomorphisms. Assume that $\mathbb{R}^{2 n}$ is decomposed as a product of two symplectic vector spaces $V$ and $W$ and $H$ is also split, i.e., $H=H_{V}+H_{W}$, where $H_{V}$ and $H_{W}$ are Hamiltonians on $V$ and, respectively, $W$ with flows fixing the origin. Assume, in addition, that the time one-map $\varphi_{H_{V}}$ of $H_{V}$ is non-degenerate and the time-one map $\varphi_{H_{W}}$ of $H_{W}$ is strongly degenerate. Then combining the previous two particular cases and applying the Künneth formula for local Floer homology (see (LF4)), we conclude that the theorem holds for $H$.

More generally, assume that $\varphi$, but not necessarily $H$, is split, i.e., $\varphi_{H}=$ $\left(\varphi_{V}, \varphi_{W}\right)$. Then $\varphi_{V}$ and $\varphi_{W}$ are the germs of symplectomorphisms fixing $p$, and hence both $\varphi_{V}$ and $\varphi_{W}$ are Hamiltonian; see Remark 4.1. As above, denote by $H_{V}$ and $H_{W}$ some Hamiltonians generating $\varphi_{V}$ and, respectively, $\varphi_{W}$. We do not necessarily have $H=H_{V}+H_{W}$, but since local Floer homology is determined by $\varphi$ up to a shift of indices,

$$
\mathrm{HF}_{*}\left(H^{\# k}, p\right)=\mathrm{HF}_{*+s_{k}^{\prime}}\left(H_{V}^{\# k}+H_{W}^{\# k}, p\right) .
$$

The Hamiltonian $H_{V}+H_{W}$ is split and, as has been shown above, the theorem holds for $H_{V}+H_{W}$. It remains to prove that the additional shifts $s_{k}^{\prime}$ are even. This, however, is clear, for $s_{k}^{\prime} / 2$ is the Maslov index of the loop obtained by taking the concatenation of the flow of $H^{\# k}$ and the inverse flow of $H_{V}^{\# k}+H_{W}^{\# k}$.

4.5. The general case. Let $\varphi$ be the germ of the Hamiltonian diffeomorphism fixing the origin $p$ in $\mathbb{R}^{2 n}$ and generated by $H$. For some decomposition $\mathbb{R}^{2 n}=V \times W$ the linearization $d \varphi_{p}$ splits as the direct sum of a symplectic linear map on $V$ whose eigenvalues are all different from one and a symplectic linear map on $W$ with all eigenvalues equal to one. Then, if $k$ is admissible, the same splitting holds for $d \varphi_{p}^{k}$. We will show that $\varphi$ is homotopic to a split map via Hamiltonian diffeomorphisms with uniformly isolated fixed point at $p$ and linearization $d \varphi_{p}$. Denote such a homotopy by $\varphi_{s}, s \in[0,1]$. Then $p$ is also a uniformly isolated fixed point for all maps in the iterated homotopy $\varphi_{s}^{k}$ (see Remark 1.2 and Proposition 7.1) and the theorem follows from Case 3 and the invariance of local Floer homology under homotopy; see (LF1).

To be more precise, let $K_{s}$ be the Hamiltonian generating $\varphi_{s}$ as its time-one map and obtained, up to an obvious reparametrization, by concatenating the flow $\varphi_{H}^{t}, t \in[0,1]$, with the homotopy $\varphi_{\zeta}, \zeta \in[0, s]$. Then $p$ is a uniformly isolated fixed point of $\varphi_{K_{s}}^{k}$ for all $s \in[0,1]$ and all admissible $k$. Hence, by (LF1),

$$
\mathrm{HF}_{*}\left(H^{\# k}, p\right)=\mathrm{HF}_{*}\left(K_{1}^{\# k}, p\right) .
$$

In addition, $\Delta_{H}(p)=\Delta_{K_{1}}(p)$, for $d \varphi_{s}$ at $p$ is constant. Since $\varphi_{1}=\varphi_{K_{1}}$ is split, the theorem holds for $K_{1}$. Therefore, the theorem also holds for $H$. 
Let us now construct the homotopy $\varphi_{s}$. Let $N_{V}$ and $N_{W}$ be Lagrangian complements to the diagonals $\Delta_{V} \subset V \times \bar{V}$ and, respectively, $\Delta_{W} \subset W \times \bar{W}$, transverse to the graphs of $\left.d \varphi_{p}\right|_{V}$ and $\left.d \varphi_{p}\right|_{W}$. Then $N=N_{V} \times N_{W}$ is a Lagrangian complement to the diagonal $\Delta$ in $\mathbb{R}^{2 n} \times \overline{\mathbb{R}}^{2 n}$, transverse to the graph of $d \varphi_{p}$, and hence to the graph of $\varphi$ on a small neighborhood of $p$. Denote by $F$ the generating function of $\varphi$ with respect to $N$ on a neighborhood of $p$. Note that $p$ is an isolated critical point of $F$ and $d^{2} F_{p}$ is split.

We will construct a family of functions $F_{s}, s \in[0,1]$, on a neighborhood of $p$ starting with $F_{0}=F$ and such that

- $p$ is a uniformly isolated critical point of $F_{s}$,

- $d^{2}\left(F_{s}\right)_{p}=d^{2} F_{p}$

- $F_{1}$ is split, i.e., $F_{1}$ is the sum of a function $q$ on $V$ and a function $f$ on $W$ near $p$.

Once the family $F_{s}$ is constructed, $\varphi_{s}$ is defined in an obvious way via identifying the graph of $\varphi_{s}$ with the graph of $d F_{s}$ in $\mathbb{R}^{2 n} \times \overline{\mathbb{R}}^{2 n}=\Delta \times$ $N=T^{*} \Delta$. (The graph of $d F_{s}$ is transverse to the fibers of the projection $\pi: \mathbb{R}^{2 n} \times \overline{\mathbb{R}}^{2 n} \rightarrow \mathbb{R}^{2 n}$ near $p$, since $d^{2}\left(F_{s}\right)_{p}=d^{2} F_{p}$ and the graph of $d F$, coinciding with the graph of $\varphi$, is transverse to the fibers.) Note also that in the decomposition $F_{1}=q+f$, the function $q$ is a non-degenerate quadratic form on $V$ (in fact, $q=\left.d^{2} F_{p}\right|_{V}$ ) and $f$ is a function on $W$ with isolated critical point at the origin.

To find the family $F_{s}$, we argue as follows. First observe that, by the implicit function theorem, there exists (near $p$ ) a unique smooth map $\Phi: W \rightarrow V$ such that $\Phi(0)=0$ and $\left.F\right|_{V \times w}$ has a critical point at $\Phi(w)$. Let $\Sigma$ be the graph of $\Phi$. It is easy to see that $d \Phi$ vanishes at the origin, for $d^{2} F_{p}$ is split, and hence $\Sigma$ is tangent to $W$ at $p$. Now $F_{s}$ is constructed in two steps. First, we use an isotopy on a neighborhood of $p$, fixing $p$ and having the identity linearization at $p$, to move $\Sigma$ to $W$. This isotopy turns $F$ into a function, say $F_{0.5}$, such that $\left.F_{0.5}\right|_{V \times w}$ has a non-degenerate critical point at $(0, w)$ for all $w$ near the origin. As the second step, we apply the parametric Morse lemma to $\left.F_{0.5}\right|_{V \times w}$ to obtain a homotopy from $F_{0.5}$ to a function $F_{1}$ of the desired form $q+f$.

This concludes the proof of the theorem.

\section{Symplectically degenerate maxima}

Strongly degenerate periodic orbits with persistent Floer homology in degree $n$, referred to in $[\mathbf{G i 2}]$ as symplectically degenerate maxima, play a particularly interesting role in the proof of the Conley conjecture; see [Gi2]. This role is further exemplified by Theorem 1.1 and we feel that features of such orbits merit further investigation. In this section, we characterize symplectically degenerate maxima in homological and geometrical terms and then, in 
Section 5.2, touch upon "vanishing properties" of the pair-of-pants product in local Floer homology. Namely, we show that a periodic orbit is a symplectically degenerate maximum if and only if the product is not in a certain sense nilpotent. The latter topic is rather tangential to the main subject of the paper and is treated here very briefly, skipping some technical details.

5.1. Homological and geometrical properties of symplectically degenerate maxima. Let $\gamma$ be a one-periodic orbit of the flow of a Hamiltonian $H$ on a symplectically aspherical manifold $M^{2 n}$. In fact, it suffices to assume that $H$ is the germ of a Hamiltonian on a neighborhood of $\gamma$.

Definition 5.1. The orbit $\gamma$ is said to be a symplectically degenerate maximum of $H$ if $\Delta_{H}(\gamma)=0$ and $\operatorname{HF}_{n}(H, \gamma) \neq 0$.

Example 5.1. Let $H$ be an autonomous Hamiltonian attaining a strict local maximum at $p$. Assume in addition that $d^{2} H_{p}=0$ or, more generally, that all eigenvalues of $d^{2} H_{p}$ are zero. Then it is easy to see that $p$ is a symplectically degenerate maximum of $H$, cf. Proposition 5.2. (Here, as is customary in Hamiltonian dynamics, the eigenvalues of a quadratic form on a symplectic vector space are, by definition, the eigenvalues of the linear symplectic vector field generated by the quadratic form.)

Proposition 5.1. The following conditions are equivalent:

(a) the orbit $\gamma$ is a symplectically degenerate maximum of $H$;

(b) $\operatorname{HF}_{n}\left(H^{\# k_{i}}, \gamma^{k_{i}}\right) \neq 0$ for some sequence of admissible iterations $k_{i} \rightarrow$ $\infty$

(c) the orbit $\gamma$ is strongly degenerate, $\operatorname{HF}_{n}(H, \gamma) \neq 0$ and $\operatorname{HF}_{n}\left(H^{\# k}, \gamma^{k}\right)$ $\neq 0$ for at least one admissible iteration $k \geq n+1$.

Proof. The facts that (a) and (b) are equivalent and that (a) implies (c) follow immediately from Theorem 1.1. To show that (c) implies (a), it is sufficient to prove that $\Delta_{H}(\gamma)=0$. Assume the contrary. Then $\left|\Delta_{H}(\gamma)\right| \geq 2$ since $\Delta_{H}(\gamma) \in 2 \mathbb{Z}$ due to the assumption that $\gamma$ is strongly degenerate and (MI8). Thus,

$$
\left|\Delta_{H \# k}\left(\gamma^{k}\right)\right|=k\left|\Delta_{H}(\gamma)\right| \geq 2 k \geq 2(n+1) .
$$

Therefore, by (LF5), $\mathrm{HF}_{*}\left(H^{\# k}, \gamma^{k}\right)$ is supported in the interval $[n+2,3 n+2]$, which contradicts the condition that $\operatorname{HF}_{n}\left(H^{\# k}, \gamma^{k}\right) \neq 0$.

As a consequence of the proposition, we observe that, for any admissible iteration $k$, the orbit $\gamma^{k}$ is a symplectically degenerate maximum if and only if $\gamma$ is a symplectically degenerate maximum. 
To illuminate the geometrical nature of symplectically degenerate maxima, let us assume that the orbit $\gamma$ is constant, i.e., $\gamma(t) \equiv p$ and $H$ is defined on a neighborhood of $p$. Then, as our next result shows, the behavior of $\varphi_{H}$ near $p$ is similar to that described in Example 5.1. The essence of this result is that $p$ is a symplectically degenerate maximum of $H$ if and only if $\varphi_{H}$ can be generated by a Hamiltonian $K$ with local maximum at $p$ and arbitrarily small Hessian.

Proposition 5.2. The point $p$ is a symplectically degenerate maximum of $H$ if and only if for every $\epsilon>0$ there exists a Hamiltonian $K$ near $p$ such that $\varphi_{K}=\varphi_{H}$ in the universal covering of the group of local symplectomorphisms fixing $p$ and

(i) $p$ is a strict local maximum of $K_{t}$ for all $t \in S^{1}$,

(ii) $\left\|d^{2}\left(K_{t}\right)_{p}\right\|_{\Xi}<\epsilon$ for all $t \in S^{1}$ and some symplectic basis $\Xi$ in $T_{p} M$.

To clarify the terminology used here, recall that $\left\|d^{2}\left(K_{t}\right)_{p}\right\|_{\Xi}$ stands for the norm of $d^{2}\left(K_{t}\right)_{p}$ with respect to the Euclidean inner product on $T_{p} M$ for which $\Xi$ is an orthonormal basis; see [Gi2, Section 2.1.3].

Proof of Proposition 5.2. The non-trivial part of the proposition is that a Hamiltonian $K$ with the required properties exists whenever $p$ is a symplectically degenerate maximum. This is established in [Gi2, Proposition 4.5]. (Note that in [Gi2, Proposition 4.5] the roles of the assertion and the hypotheses of Proposition 5.2 above are interchanged, cf. [Gi2, Definition 4.1].) Conversely, $\operatorname{HF}_{*}(H, p)=\operatorname{HF}_{*}(K, p)$ by (LF3) and $\Delta_{H}(p)=\Delta_{K}(p)$ by (MI8). We infer from (ii) that $\left|\Delta_{K}(p)\right|$ can be made arbitrarily small for a suitable choice of $K$. Thus, $\Delta_{H}(p)=0$. Furthermore, using (i), it is straightforward to construct a $C^{2}$-small perturbation $\tilde{K}$ of $K$ such that $p$ is a non-degenerate local maximum of $\tilde{K}_{t}$, and $\tilde{K}$ has no other oneperiodic orbits near $p$. Since $\left\|d^{2}\left(\tilde{K}_{t}\right)_{p}\right\|_{\Xi}$ is small, $\mu_{\mathrm{CZ}}(\tilde{K}, p)=n$. Hence, $\operatorname{HF}_{n}(H, p)=\operatorname{HF}_{n}(\tilde{K}, p)=\mathbb{Z}_{2}$.

Remark 5.1. It is clear from Propositions 5.1 and 5.2 that the definition of a symplectically degenerate maximum given here is equivalent to the one in [Gi2]. (As a consequence, the additional requirement (K3) in [Gi2, Definition 4.1] is superfluous and follows from (K1) and (K2), reformulations of (i) and (ii).) The proof of Proposition 5.2 also shows that in Definition 5.1 and in (b) and (c) the conditions $\operatorname{HF}_{n}(H, \gamma) \neq 0$ and $\operatorname{HF}_{n}\left(H^{\# k}, \gamma^{k}\right) \neq 0$ can be replaced by the more specific requirement that these Floer homology groups are isomorphic to $\mathbb{Z}_{2}$.

The definition of a symplectically degenerate maximum and Propositions 5.1 and 5.2 extend word-for-word to isolated fixed points of Hamiltonian diffeomorphisms $\varphi: M \rightarrow M$, for the local Floer homology and the mean index are completely determined by $\varphi$. When $\varphi$ is just the germ of a Hamiltonian 
diffeomorphism near an isolated fixed point $p$, the grading of local Floer homology and the mean index are defined only up to a shift by the same even integer. In this case, we say that $p$ is a local symplectically degenerate maximum when $\varphi$ can be generated by a Hamiltonian $H$ with flow fixing $p$ and symplectically degenerate maximum at $p$. By (MI8), (LF5) and (LF6), this is equivalent to that $\Delta_{K}(p) \in \mathbb{Z}$ and $\operatorname{HF}_{n+\Delta_{K}(p)}(K, p) \neq 0$ for any (or, equivalently, some) Hamiltonian $K$ with $\varphi_{K}=\varphi$ and $\varphi_{K}^{t}(p) \equiv p$. Furthermore, then $\Delta_{K}(p)$ is necessarily even. (Warning: a fixed point $p$ of $\varphi: M \rightarrow M$ can be a local symplectically degenerate maximum of the germ of $\varphi$ at $p$, but not a symplectically degenerate maximum of $\varphi$.)

5.2. Product in local Floer homology. The construction of the pair-ofpants product in Floer homology (see, e.g., [MS, PSS, Sc2]) carries over in an obvious way to local Floer homology. Thus, we have a product

$$
\underbrace{\operatorname{HF}_{*}(H, \gamma) \otimes \ldots \otimes \operatorname{HF}_{*}(H, \gamma)}_{r} \rightarrow \operatorname{HF}_{*}\left(H^{\# r}, \gamma^{r}\right),
$$

where $\gamma$ is an isolated one-periodic orbit of $H$ and $r$ is admissible. When $u_{i} \in \operatorname{HF}_{l_{i}}(H, \gamma)$, the product $u_{1} \cdots \cdot u_{r}$ has degree $l_{1}+\cdots+l_{r}-(r-1) n$, where $\operatorname{dim} M=2 n$. Up to a shift of degree, the product is a feature of the germ of $\varphi=\varphi_{H}$ at the fixed point $\gamma(0)=p$. Indeed, assume for the sake of simplicity that $\gamma$ is constant. Then $\mathrm{HF}_{*}\left(H^{\# r}, p\right)$ is isomorphic to $\mathrm{HF}_{*+m_{r}}\left(K^{\# r}, p\right)$ for any two Hamiltonians $H$ and $K$ generating $\varphi$ near $p$ and some $m_{r}$. The isomorphism is induced by the composition with the corresponding loop of Hamiltonian diffeomorphisms near $p$ and, as is clear from the definition, this isomorphism preserves the pair-of-pants product.

To set the stage for our discussion of "vanishing properties" of the pairof-pants product in local Floer homology, recall that the Morse theoretic counterpart of this product is the cup product in local Morse homology; see, e.g., $[\mathbf{J o}, \mathbf{S c 2}]$. Let $F$ be a germ of a smooth function near its isolated critical point $p \in \mathbb{R}^{m}$. One can show that the cup product in $\operatorname{HM}_{*}(F, p)$ is trivial unless $p$ is a local maximum of $F$. (In the latter case, $\operatorname{HM}_{*}(F, p)$ is concentrated in degree $m$ and $u^{k}=u$ for all $k$, where $u$ is the generator of $\operatorname{HM}_{m}(F, p)=\mathbb{Z}_{2}$.) In particular, $u \cdot v=0$ for any two distinct elements $u$ and $v$ in $\operatorname{HM}_{*}(F, p)$ regardless of whether $p$ is a local maximum or not. These properties are inherited, in a somewhat weaker form, by the pair-of-pants product.

Proposition 5.3. Assume that $\gamma(0)$ is not a local symplectically degenerate maximum of the germ of $\varphi_{H}$ at $\gamma(0)$. Then the product in $\mathrm{HF}_{*}(H, \gamma)$ is "nilpotent", i.e., there exists $r_{0}$, depending only on the linearized flow along $\gamma$, such that $u_{1} \cdot \ldots \cdot u_{r}=0$ for any admissible $r \geq r_{0}$ and any classes $u_{1}, \ldots, u_{r}$ in $\operatorname{HF}_{*}(H, \gamma)$. 
Proof. The proof is essentially the observation that, unless $\gamma(0)$ is a local symplectically degenerate maximum, the degree $l$ of $u_{1} \cdot \ldots \cdot u_{r}$ is necessarily outside the support of $\mathrm{HF}_{*}\left(H^{\# r}, \gamma^{r}\right)$ for a large enough $r$. Let, as above, $u_{i} \in \operatorname{HF}_{l_{i}}(H, \gamma)$ and $u_{i} \neq 0$. Then the mean $\left(l_{1}+\ldots+l_{r}\right) / r$ also lies in the support of $\mathrm{HF}_{*}(H, \gamma)$, which, in turn, is contained in $\left(-\infty, \Delta_{H}(\gamma)+n\right)$, since $\gamma(0)$ is not a local symplectically degenerate maximum of the germ of $\varphi_{H}$. Thus,

$$
\left(l_{1}+\cdots+l_{r}\right) / r-\Delta_{H}(\gamma)-n \leq-\delta
$$

for some $\delta>0$ independent of $r$ and $l_{1}, \ldots, l_{r}$. It follows that

$$
\begin{aligned}
l-r \Delta_{H}(\gamma) & =l_{1}+\cdots+l_{r}-(r-1) n-r \Delta_{H}(\gamma) \\
& =r\left(\frac{l_{1}+\cdots+l_{r}}{r}-\Delta_{H}(\gamma)-n\right)+n \\
& \leq-\delta r+n .
\end{aligned}
$$

The support of $\mathrm{HF}_{*}\left(H^{\# r}, \gamma^{r}\right)$ is contained in $\left[r \Delta_{H}(\gamma)-n, r \Delta_{H}(\gamma)+n\right]$. Hence, when $r>2 n / \delta$, the degree $l$ of the product is outside the support.

In Proposition 5.3, the assumption that $\gamma(0)$ is not a local symplectically degenerate maximum is essential as the following example shows.

Example 5.2. Assume that $p$ is a strict local maximum of an autonomous Hamiltonian $H$ and the Hessian of $H$ at $p$ is identically zero. Then $\mathrm{HF}_{*}\left(H^{\# k}, p\right)=\mathrm{HM}_{*+n}(H, p)$ for every $k$ and the isomorphism intertwines the pair-of-pants product and the cup product; cf. Example 3.4. (This is essentially the fact that the pair-of-pants product in Floer homology of a $C^{2}$-small autonomous Hamiltonian is equal to the cup product in its Morse homology, [Sc2]; see Section 3.3.) Hence, denoting by $u$ the generator of $\operatorname{HF}_{n}(H, p)=\mathbb{Z}_{2}$, we see that $u^{k} \neq 0$ for any $k$ and, moreover, $u^{k}$ is the generator of $\operatorname{HF}_{n}\left(H^{\# k}, p\right)=\mathbb{Z}_{2}$. Replacing the requirement that $d^{2} H_{p}=0$ by the condition that the Hessian is small, we also note that the pair-of-pants product can be non-trivial even if $p$ is non-degenerate.

A slightly more elaborate version of the argument from this example proves that $u^{k}$ is a generator of $\operatorname{HF}_{n}\left(H^{\# k}, \gamma\right)=\mathbb{Z}_{2}$ for any symplectically degenerate maximum and the same is true (up to a shift of degree) for local symplectically degenerate maxima. (Namely, reasoning as in Section 4.3 and using Lemma 3.1, one can equate the local Floer homology of $H$ and its iterations to the Morse homology of a generating function with a strict, nearly degenerate maximum at $p$. Similarly to the case of an autonomous Hamiltonian, the resulting isomorphism intertwines products.) This leads to a variety 
of characterizations of symplectically degenerate maxima via the pair-ofpants product. For instance, it then follows from Proposition 5.3 that $\gamma$ is a symplectically degenerate maximum of $H$ if and only if $\operatorname{HF}_{n}(H, p)=\mathbb{Z}_{2}$ and $u^{k} \neq 0$, where $u$ is the generator of $\operatorname{HF}_{n}(H, p)$, for every admissible iteration $k$.

Instances where the pair-of-pants product vanishes are not exhausted by Proposition 5.3. For example, arguing as in the proof of Theorem 1.1, one can show that $u \cdot v=0$ for any two distinct elements $u$ and $v$ in $\operatorname{HF}_{*}(H, \gamma)$.

Remark 5.2. One may also consider products of the form $w_{1} \cdot \ldots \cdot w_{r} \in$ $\operatorname{HF}_{l}\left(H^{\# k}, \gamma^{k}\right)$ with $w_{i} \in \mathrm{HF}_{l_{i}}\left(H^{\# k_{i}}, \gamma^{k_{i}}\right)$, where $l=l_{1}+\cdots+l_{r}-(r-1) n$ as above and $k=k_{1}+\cdots+k_{r}$. Properties of such products are more involved than those of the products with $k_{1}=\ldots=k_{r}=1$ considered above. For instance, we do not assert that Proposition 5.3 holds for $w_{1} \cdot \ldots \cdot w_{r}$ and it is certainly not true that the product of two such distinct elements $w_{1}$ and $w_{2}$ is necessarily zero. However, Proposition 5.3 readily extends to products of this form when all iterations $k_{i}$ are bounded from above.

In conclusion note that Proposition 5.3 is analogous to the nilpotence results for the Chas-Sullivan product established in [GoHi]. In fact, it is not unreasonable to expect the corresponding local homology groups and products to be isomorphic; cf. $[\mathbf{A S 1}, \mathbf{A S 2}, \mathbf{S W}, \mathbf{V i 2}]$ and references therein.

\section{Proof of Theorem 1.2}

The proof of Theorem 1.2 is based on the analysis of two cases, similarly to the argument from [Gi2] establishing the Conley conjecture. Namely, since $\operatorname{HF}_{n}(H) \neq 0$, there exists a one-periodic orbit $x$ of $H$ with $\operatorname{HF}_{n}(H, x) \neq$ 0 . Thus, $\Delta_{H}(x) \geq 0$ by (LF5). The first, "non-degenerate", case is where $\Delta_{H}(x)>0$, while in the second, "degenerate", case $\Delta_{H}(x)=0$, i.e., $x$ is a symplectically degenerate maximum. Note that since, in general, $x$ is not unique, the two cases are not mutually exclusive for a given Hamiltonian $H$. Furthermore, we emphasize that here, as is required in Theorem 1.2, $M$ is assumed to be closed and symplectically aspherical.

6.1. Stability of Floer homology. In the proof, we will need the following simple observation asserting that filtered Floer homology is stable, i.e., cannot be destroyed by a relatively small perturbation of the Hamiltonian, cf. $[\mathbf{B C}, \mathbf{C h}]$. Let $K$ and $F$ be Hamiltonians on $M$. Set

$$
E^{+}=\int_{0}^{1} \max _{M} F_{t} d t \quad \text { and } \quad E^{-}=-\int_{0}^{1} \min _{M} F_{t} d t
$$


so that $\|F\|=E^{+}+E^{-}$is the Hofer energy of $F$. Furthermore, let

$$
E_{0}^{+}=\max \left\{E^{+}, 0\right\} \quad \text { and } E_{0}^{-}=\max \left\{E^{-}, 0\right\} \quad \text { and } \quad E_{0}(F)=E_{0}^{+}+E_{0}^{-} \text {. }
$$

Then

- $\mathrm{HF}_{*}^{(a+E, b+E)}(K \# F) \neq 0$ for any interval $(a, b)$ and any non-negative constant $E \geq E_{0}(F)$, whenever the natural "quotient-inclusion" map

$$
\kappa: \mathrm{HF}_{*}^{(a, b)}(K) \rightarrow \mathrm{HF}_{*}^{(a+2 E, b+2 E)}(K)
$$

is non-zero.

This fact is an immediate consequence of commutativity of the following diagram:

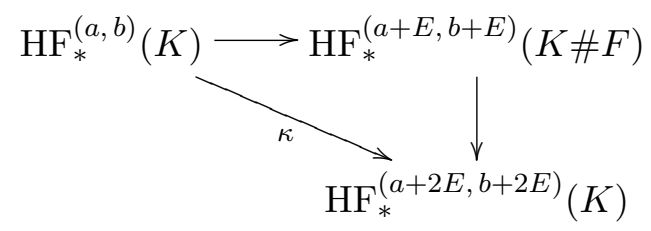

where the horizontal arrow is induced by the linear homotopy from $K$ to $K \# F$ and the vertical arrow is induced by the linear homotopy from $K \# F$ to $K$; see, e.g., [Gi1].

Remark 6.1. This stability result is admittedly very crude and can be refined in a number of ways. For instance, as is clear from its proof, the intervals $(a+E, b+E)$ and $(a+2 E, b+2 E)$ can be replaced by the intervals $\left(a+E_{0}^{+}, b+E_{0}^{+}\right)$and, respectively, $\left(a+E_{0}, b+E_{0}\right)$. However, the present version of stability lends itself conveniently for the proof of Theorem 1.2 and affords some notational simplifications, while a more precise statement appears to only result in a marginally sharper upper bound on the action-index gap.

6.2. The "non-degenerate" case: $\operatorname{HF}_{n}(H, x) \neq 0$ and $\Delta_{H}(x)>0$. We deal with this case under somewhat less restrictive assumptions that $\operatorname{HF}_{*}(H, x) \neq 0$ and $\Delta_{H}(x)>0$. Then, as is easy to see, within every infinite set of admissible iterations there exists an infinite subsequence $l_{1}<l_{2}<\cdots$ such that

$$
\check{l} \leq l_{i+1}-l_{i} \leq \hat{l},
$$

where $\check{l}$ and $\hat{l}$ are independent of $i$ and

$$
\frac{2 n}{\Delta_{H}(x)}<\check{l} .
$$

From Theorem 1.1 it follows that the local Floer homology $\operatorname{HF}_{*}\left(H^{\# l_{i}}, x^{l_{i}}\right)$ is non-trivial and, by (LF5), supported in the interval $\left(l_{i} \Delta_{H}(x)-n\right.$, $\left.l_{i} \Delta_{H}(x)+n\right)$. As a consequence, the groups $\operatorname{HF}_{*}\left(H^{\# l_{j}}, x^{l_{j}}\right)$ and $\operatorname{HF}_{*}\left(H^{\# l_{i}}\right.$, $\left.x^{l_{i}}\right)$ have disjoint support when $j \neq i$. 
Adding a constant to $H$, we can assume without loss of generality that $A_{H}(x)=0$, and hence $A_{H \# k}\left(x^{k}\right)=0$ for all $k$. Set

$$
E:=\max _{r=1, \ldots, l} E_{0}\left(H^{\# r}\right)
$$

and let $(a, b)$ be an arbitrary interval containing zero and such that $(a+$ $2 E, b+2 E)$ also contains zero, i.e., $a+2 E<0<b$.

The sequence $\nu_{j}$ is picked as a subsequence of $l_{i}$, skipping at most every second term. Assume that $\nu_{1}, \ldots, \nu_{j-1}=l_{i-1}$ and the periodic orbits $y_{1}, \ldots, y_{j-1}$ have been chosen. Our goal is to find a $\nu_{j}$-periodic orbit $y=y_{j}$ with either $\nu_{j}=l_{i}$ or $\nu_{j}=l_{i+1}$ satisfying the requirements of Theorem 1.2. (The first orbit $y_{1}$ and the period $\nu_{1}$ equal to $l_{1}$ or $l_{2}$ are chosen in a similar fashion.)

Fix $m$ such that $\operatorname{HF}_{m}\left(H^{\# l_{i}}, x^{l_{i}}\right) \neq 0$. By (LF5),

$$
\left|m-\Delta_{H^{\# l_{i}}}\left(x^{l_{i}}\right)\right| \leq n .
$$

Under the above assumptions, $y_{j}$ and $\nu_{j}$ are chosen differently in each of the following three cases.

Case 1: $\mathrm{HF}_{m}^{(a, b)}\left(H^{\# l_{i}}\right)=0$. It is easy to see that in this case $H$ has an $l_{i}$-periodic orbit $y$, canceling the contribution of $\mathrm{HF}_{m}\left(H^{\# l_{i}}, x^{l_{i}}\right)$ to $\mathrm{HF}_{m}^{(a, b)}\left(H^{\# l_{i}}\right)$, such that $\left|\Delta_{H^{\# l_{i}}}(y)-m\right| \leq n+1$ and the action $A_{H^{\# l_{i}}}(y)$ is in the interval $(a, b)$. Furthermore, $A_{H^{\# l_{i}}}(y) \neq A_{H^{\# l_{i}}}\left(x^{l_{i}}\right)=0$ due to the choice of $y$ as an orbit canceling the contribution of $x$. Set $\nu_{j}=l_{i}$ and $y_{j}=y$. It is clear that the action and index gaps for $x^{l_{i}}$ and $y$ are bounded from above by $\max \{|a|, b\}$ and, respectively, $2 n+1$ and the action gap is strictly positive.

Case 2: $\operatorname{HF}_{m}^{(a+E, b+E)}\left(H^{\left.\# l_{i+1}\right)} \neq 0\right.$. Under this assumption, there exists an $l_{i+1}$-periodic orbit $y$ with action in the interval $(a+E, b+E)$ and $\left|\Delta_{H}^{\# l_{i+1}}(y)-m\right| \leq n$. We set $\nu_{j}=l_{i+1}$ and $y_{j}=y$. To verify the requirements of the theorem, we first note that, since $A_{H}(x)=0$, we have

$$
\left|A_{H^{\# l_{i+1}}}\left(x^{l_{i+1}}\right)-A_{H^{\# l_{i+1}}}(y)\right|=\left|A_{H^{\# l_{i+1}}}(y)\right| \leq \max \{|a+E|, b+E\},
$$

Furthermore, as is easy to check,

$$
\check{l} \Delta_{H}(x)-2 n \leq\left|\Delta_{H}^{\# l_{i+1}}\left(x^{l_{i+1}}\right)-\Delta_{H}^{\# l_{i+1}}(y)\right| \leq \hat{l} \Delta_{H}(x)+2 n .
$$

The latter inequalities give lower and upper bounds on the difference of the mean indices and, by (6.1), show that this difference is non-negative. (This is the only case where we cannot guarantee that the action gap is strictly positive.)

Case 3: $\operatorname{HF}_{m}^{(a, b)}\left(H^{\# l_{i}}\right) \neq 0$, but $\operatorname{HF}_{m}^{(a+E, b+E)}\left(H^{\# l_{i+1}}\right)=0$. First note that

$$
H^{\# l_{i+1}}=H^{\# l_{i}} \# F, \quad \text { where } \quad F=H^{\#\left(l_{i+1}-l_{i}\right)}, \quad \text { and } \quad E \geq E_{0}(F) .
$$


Here the last inequality follows from the definitions of $\hat{l}$ and $E$. Indeed, $\hat{l} \geq l_{i+1}-l_{i}$ and thus $E \geq E_{0}\left(H^{\#\left(l_{i+1}-l_{i}\right)}\right)=E_{0}(F)$.

Using stability of filtered Floer homology as in Section 6.1 with $K=H^{\# l_{i}}$ and $F=H^{\#\left(l_{i+1}-l_{i}\right)}$, we see that the quotient-inclusion map

$$
\kappa: \operatorname{HF}_{m}^{(a, b)}\left(H^{\# l_{i}}\right) \rightarrow \operatorname{HF}_{m}^{(a+2 E, b+2 E)}\left(H^{\# l_{i}}\right)
$$

is necessarily zero, for $\operatorname{HF}_{m}^{(a+E, b+E)}\left(H^{\# l_{i+1}}\right)=0$. Since $\operatorname{HF}_{m}^{(a, b)}\left(H^{\# l_{i}}\right) \neq 0$, we infer by a simple exact sequence argument that $\mathrm{HF}_{m}^{(a, a+2 E)}\left(H^{\# l_{i}}\right) \neq 0$ or/and $\mathrm{HF}_{m+1}^{(b, b+2 E)}\left(H^{\# l_{i}}\right) \neq 0$. In the former case, there exists an $l_{i}$-periodic orbit $y$ with action in the range $(a, a+2 E)$ and $\left|m-\Delta_{H \# l_{i}}(y)\right| \leq n$. In the latter case, there exists an $l_{i}$-periodic orbit $y$ with action in the range $(b, b+2 E)$ and $\left|m+1-\Delta_{H \# l_{i}}(y)\right| \leq n$. We set $\nu_{j}=l_{i}$ and $y_{j}=y$. Then

$$
0<\min \{|a+2 E|, b\}<\left|A_{H^{\# l_{i}}}(y)\right| \leq \max \{|a|, b+2 E\}
$$

and

$$
\left|\Delta_{H \# l_{i}}\left(x^{l_{i}}\right)-\Delta_{H \# l_{i}}(y)\right| \leq 2 n+1 .
$$

Combining the three cases above, it is immediate to see that the constants $e$ and $\delta$ from the statement of the theorem are then given by

$$
e=\max \{|a|, b+2 E\} \quad \text { and } \quad \delta=\max \left\{2 n+1,2 n+\hat{l} \Delta_{H}(x)\right\}
$$

and that the index gap or the action gap is necessarily positive. This completes the proof of the theorem in the "non-degenerate" case.

6.3. The "degenerate" case: $\operatorname{HF}_{n}(H, x) \neq 0$ and $\Delta_{H}(x)=0$. This is the case where $x$ is a symplectically degenerate maximum of $H$. By Theorem $1.1, x$ is strongly degenerate (thus every $k$ is admissible for $x$ ) and $\operatorname{HF}_{n}\left(H^{\# k}, x^{k}\right) \neq 0$ for all $k \geq 1$. Furthermore, Propositions 4.5 and 4.7 from [Gi2] assert that for every $\epsilon>0$ there exists an integer $k_{\epsilon}>0$ such that for all $k>k_{\epsilon}$ we have

$$
\mathrm{HF}_{n+1}^{(k c, k c+\epsilon)}\left(H^{\# k}\right) \neq 0,
$$

where $c=A_{H}(x)$. Hence, $\varphi$ has a $k$-periodic orbit $z_{k}$ with

$$
0<\left|A_{H \# k}\left(x^{k}\right)-A_{H \# k}\left(z_{k}\right)\right|<\epsilon
$$

and

$$
1 \leq\left|\Delta_{H \# k}\left(x^{k}\right)-\Delta_{H \# k}\left(z_{k}\right)\right| \leq 2 n+1 .
$$

Thus, given a quasi-arithmetic sequence of admissible iterations $l_{i}$, we can take as $\nu_{j}$ the "tail" of this sequence, i.e., its subsequence formed by $l_{i}>k_{\epsilon}$, and set $y_{j}=z_{\nu_{j}}$.

This completes the proof of Theorem 1.2. 


\section{Persistence of isolation}

The main objective of this section, which is independent of the rest of the paper, is to prove Proposition 1.1 asserting that an isolated fixed point of a diffeomorphism remains isolated under admissible iterations. In fact, we establish the following slightly more general result:

Proposition 7.1. Let $p \in M$ be a fixed point of a family of $C^{1}$-smooth diffeomorphisms $\varphi_{s}: M \rightarrow M$ with $s \in[0,1]$ and let $k$ be an admissible iteration of $\varphi_{s}$ (for all $\left.s\right)$ with respect to $p$. Then, for any $s$, every $k$-periodic point of $\varphi_{s}$ in a sufficiently small neighborhood of $p$ (depending on $k$, but not on $s)$ is a fixed point of $\varphi_{s}$.

When $\varphi_{s}$ is independent of $s$, i.e., $\varphi_{s} \equiv \varphi$, and $p$ is isolated, this result turns into Proposition 1.1. When $p$ is uniformly isolated, we obtain the parametric version of Proposition 1.1 stated in Remark 1.2.

Proof. Since the problem is local, we can assume without lost of generality that $M=\mathbb{R}^{m}$ and $p=0$. Fixing an admissible iteration $k$, we need to show that every $k$-periodic point of $\varphi_{s}$ sufficiently close to $p$ is a fixed point, i.e., every fixed point of $\varphi_{s}^{k}$ near $p$ is in fact a fixed point of $\varphi_{s}$.

We start with an observation of a general nature. Let $\xi$ be a map $\mathbb{Z}_{k} \rightarrow$ $\mathbb{R}^{m}$. Set $\dot{\xi}_{l}=\xi_{l+1}-\xi_{l}$, where $\xi_{l}=\xi(l)$ and $l \in \mathbb{Z}_{k}$, and $\|\xi\|_{L^{1}}:=\left\|\xi_{1}\right\|+\ldots+$ $\left\|\xi_{k}\right\|$. (Here, $\left\|\xi_{i}\right\|$ denotes the norm of the vector $\xi_{i} \in \mathbb{R}^{m}$.) Thus, $\dot{\xi}$ is again a map $\mathbb{Z}_{k} \rightarrow \mathbb{R}^{m}$ and $\|\cdot\|_{L^{1}}$ is a norm on the linear space of maps $\xi$. We claim that

$$
\|\xi\|_{L^{1}} \leq c(k)\|\dot{\xi}\|_{L^{1}} \text { whenever } \xi \text { has zero mean, i.e., } \xi_{1}+\cdots+\xi_{k}=0,
$$

where the constant $c(k)$ depends only on $k$ and $m$. Indeed, $1 / c(k)$ is the minimum of the function $\xi \mapsto\|\dot{\xi}\|_{L^{1}}$ on the $\|\cdot\|_{L^{1} \text {-unit sphere in the linear }}$ space of all maps $\xi$ with zero mean. It is clear that this minimum is strictly positive, and hence $c(k)$ is finite. (The choice of the norm in (7.1) effects only the numerical value of $c(k)$, which is immaterial for our purposes.)

To illustrate the idea of the proof, let us first consider, as an example, a particular case of the proposition.

Example 7.1. Assume that $\varphi_{s} \equiv \varphi$ is independent of $s$. Furthermore, assume that $d \varphi_{p}=\mathrm{id}$, i.e., $\varphi=\mathrm{id}+f$, where $d f_{p}=0$ and hence $\|f\|_{C^{1}}$ is small on a small neighborhood of $p$. We claim that a $k$-periodic orbit $z=\left\{z_{1}, \ldots, z_{k}\right\}$ of $\varphi$ is necessarily a fixed point of $\varphi$, whenever $z$ is close to $p$. Indeed, $\dot{z}_{l}=f\left(z_{l}\right)$ and $\left\|\ddot{z}_{l}\right\|=\left\|f\left(z_{l+1}\right)-f\left(z_{l}\right)\right\| \leq\|f\|_{C^{1}} \cdot\left\|\dot{z}_{l}\right\|$. Hence,

$$
\|\ddot{z}\|_{L^{1}} \leq\|f\|_{C^{1}} \cdot\|\dot{z}\|_{L^{1}} .
$$

Let the neighborhood containing the orbit $z$ be so small that $\|f\|_{C^{1}}<c(k)^{-1}$. Then, applying (7.1) with $\xi=\dot{z}$, we conclude that $\dot{z}=0$. In other words, $z$ 
is a constant $k$-periodic orbit, i.e., a fixed point, of $\varphi$. (This argument is a discrete version the Yorke period estimate, [Yo]; cf. [HZ, pp. 184-185].)

The proof of the general case is essentially a combination of the argument from Example 7.1 and of an application of the inverse function theorem.

First note that by compactness of $[0,1]$ it suffices to prove the result for $s$ in an arbitrarily small neighborhood $I$ of $s_{0} \in[0,1]$. If one is not an eigenvalue of $d\left(\varphi_{s_{0}}\right)_{p}$, the same is true for $d\left(\varphi_{s_{0}}^{k}\right)_{p}$ since $k$ is admissible, and the assertion follows from the inverse function theorem. Thus, we can assume that $\lambda=1$ is among the eigenvalues. Denote by $S_{\rho}$ the circle of radius $\rho>0$ centered at one. Let $\rho>0$ be so small that the only eigenvalue of $d\left(\varphi_{s_{0}}\right)_{p}$ within $S_{\rho}$ is one and, moreover, the same is true for $d\left(\varphi_{s_{0}}^{k}\right)_{p}$, i.e., $\lambda^{k}$ is outside $S_{\rho}$ for every eigenvalue $\lambda \neq 1$.

Let us decompose $\mathbb{R}^{m}$ as $V(s) \times W(s)$ so that the linearization $d\left(\varphi_{s}\right)_{p}$ splits as the direct sum of a linear map on $V(s)$ whose eigenvalues are outside $S_{\rho}$ and a linear map on $W(s)$ with all eigenvalues within $S_{\rho}$. Then $k$ is admissible for all $\varphi_{s}$ with $s$ in a small neighborhood $I$ of $s_{0}$ (depending on $\rho$ ), and the spaces $V(s)$ and $W(s)$ have constant dimensions and depend smoothly on $s$. Hence, conjugating $\varphi$ by a linear transformation (smooth in $s)$, we can make $V(s)$ and $W(s)$ independent of $s$. Set $V=V(s)$ and $W=W(s)$. The splitting of $d\left(\varphi_{s}\right)_{p}$ gives rise to the splitting of $d\left(\varphi_{s}^{k}\right)_{p}$ and, once $\rho>0$ and $I$ are sufficiently small, all eigenvalues of $\left.d\left(\varphi_{s}^{k}\right)_{p}\right|_{W}$ are within $S_{\rho}$ while all eigenvalues of $\left.d\left(\varphi_{s}^{k}\right)_{p}\right|_{V}$ are outside $S_{\rho}$. In particular, id $-\left.d\left(\varphi_{s}^{k}\right)_{p}\right|_{V}$ is invertible.

In what follows, we will denote $\varphi_{s}$ by $\varphi$ suppressing the superscript $s$ and assuming that $s$ is in a neighborhood $I$ of $s_{0}$ and that $\rho>0$ and $I$ are as small as necessary.

Let $\left(v_{0}, w_{0}\right)$ and $\left(v_{1}, w_{1}\right)$ be $k$-periodic points of $\varphi$ in the ball $B(r) \subset V \times W$ of radius $r$, centered at the origin $p$. Then

$\left\|v_{1}-v_{0}\right\| \leq C(r)\left\|w_{1}-w_{0}\right\|, \quad$ where $C(r) \rightarrow 0$ uniformly in $s \in I$ as $r \rightarrow 0$.

To see this, note that for any fixed point $(v, w)$ of $\varphi^{k}$, we must have $v=\psi_{k}(v, w)$, where $\psi_{k}$ the $V$-component of $\varphi^{k}$. The linearization of id $-\psi_{k}(\cdot, 0)$ at the origin $p$ is non-singular, for $k$ is admissible. Thus, by the implicit function theorem, there exists a unique smooth map $w \mapsto v(w)$ on a neighborhood of the origin in $W$, solving the equation $v=\psi_{k}(v, w)$. In particular, $v_{0}=v\left(w_{0}\right)$ and $v_{1}=v\left(w_{1}\right)$. Furthermore, using the fact that $d \varphi_{p}^{k}$ is split, it is easy to show that the linearization $D v_{p}$ of this map at the origin $p$ is identically zero. Hence, $C(r)=\|D v\|_{C^{0}(B(r))} \rightarrow 0$ as $r \rightarrow 0$ (uniformly in $s$ ), and (7.2) follows.

Let us set $\varphi(v, w)=\left(\psi_{w}(v), \eta_{v}(w)\right)$, where $v \in V$ and $w \in W$. Here, we view the $V$-component $\psi$ of $\varphi$ as a family of maps $V \rightarrow V$ parametrized 
by $w \in W$ and, likewise, the $W$-component $\eta$ is a family of maps $W \rightarrow W$ parametrized by $V$.

Since $\left.d \varphi_{p}\right|_{W}$ has all eigenvalues within the $\rho$-neighborhood of one, $\left.d \varphi_{p}\right|_{W}$ can be made close to the identity, up to an error of order $O(\rho)$, by conjugating $\varphi$ by a linear map depending smoothly on $s$. As a consequence, we may assume without loss of generality that $\eta_{v}$ is arbitrarily $C^{1}$-close to id on a small neighborhood $B_{W}$ of $0 \in W$ for all $v$ in some ball $B_{V}$ centered at $0 \in V$. Setting $\eta_{v}=\mathrm{id}+f_{v}$, we chose $\rho$, the interval $I$, the conjugation, and the balls $B_{W}$ and $B_{V}$ so that

$$
\max _{v \in B_{V}}\left\|f_{v}\right\|_{C^{1}} \leq \frac{1}{2 c(k)}
$$

Let $z=\left(v_{0}, w_{0}\right)$ be a $k$-periodic point of $\varphi$ and let $\left(v_{l}, w_{l}\right)=z_{l}=\varphi^{l}(z)$. Our next goal is to show that $w_{l}=w_{0}$ for all $l \in \mathbb{Z}_{k}$, provided that $z$ is sufficiently close to the origin. Without loss of generality, we may assume that $z_{l} \in B(r) \subset B_{V} \times B_{W}$ for all $l$. By definition,

$$
\left\{\begin{array}{l}
v_{l+1}=\psi_{w_{l}}\left(v_{l}\right), \\
w_{l+1}=w_{l}+f_{v_{l}}\left(w_{l}\right) .
\end{array}\right.
$$

Thus, $\dot{w}_{l+1}=w_{l+1}-w_{l}=f_{v_{l}}\left(w_{l}\right)$ and

$$
\begin{aligned}
\ddot{w}_{l+1}=\dot{w}_{l+1}-\dot{w}_{l}= & f_{v_{l}}\left(w_{l}\right)-f_{v_{l-1}}\left(w_{l-1}\right) \\
= & f_{v_{l}}\left(w_{l}\right)-f_{v_{l}}\left(w_{l-1}\right) \\
& +f_{v_{l}}\left(w_{l-1}\right)-f_{v_{l-1}}\left(w_{l-1}\right) .
\end{aligned}
$$

Therefore,

$$
\left\|\ddot{w}_{l+1}\right\| \leq\left\|f_{v_{l}}\left(w_{l}\right)-f_{v_{l}}\left(w_{l-1}\right)\right\|+\left\|f_{v_{l}}\left(w_{l-1}\right)-f_{v_{l-1}}\left(w_{l-1}\right)\right\| .
$$

Clearly,

$$
\left\|f_{v_{l}}\left(w_{l}\right)-f_{v_{l}}\left(w_{l-1}\right)\right\| \leq\left\|f_{v_{l}}\right\|_{C^{1}} \cdot\left\|\dot{w}_{l}\right\|
$$

and, for some constant $C$ independent of $s$, we obtain using (7.2) that

$$
\begin{aligned}
\left\|f_{v_{l}}\left(w_{l-1}\right)-f_{v_{l-1}}\left(w_{l-1}\right)\right\| & \leq\left\|f_{v_{l}}-f_{v_{l-1}}\right\|_{C^{0}} \\
& \leq C \cdot\left\|v_{l}-v_{l-1}\right\| \\
& \leq C \cdot C(r) \cdot\left\|\dot{w}_{l}\right\| .
\end{aligned}
$$

Combining these inequalities, we see that $\left\|\ddot{w}_{l+1}\right\| \leq\left(\left\|f_{v_{l}}\right\|_{C^{1}}+C \cdot C(r)\right)\left\|\dot{w}_{l}\right\|$, and hence

$$
\|\ddot{w}\|_{L^{1}} \leq\left(\max _{v \in B_{V}}\left\|f_{v}\right\|_{C^{1}}+C \cdot C(r)\right)\|\dot{w}\|_{L^{1}} .
$$

Therefore, by (7.3), once $r$ is so small that $C \cdot C(r)<c(k)^{-1} / 2$, we have either $\|\ddot{w}\|_{L^{1}}<c(k)^{-1}\|\dot{w}\|_{L^{1}}$ or $\dot{w} \equiv 0$. On the other hand, (7.1) applied to $\xi=\dot{w}$, yields that $\|\ddot{w}\|_{L^{1}} \geq c(k)^{-1}\|\dot{w}\|_{L^{1}}$. Thus, as in Example 7.1, $\dot{w} \equiv 0$, and hence $w_{0}=\ldots=w_{1}$. 
It remains to show that $v_{1}=\ldots=v_{k}$, for then $z=\left(v_{0}, w_{0}\right)$ is a fixed point of $\varphi$. Note that $v_{1}, \ldots, v_{k}$ is a $k$-periodic orbit of $\psi_{w_{0}}$ lying in $V \times w_{0}$. By the inverse function theorem, $\psi_{w}$ has a unique non-degenerate fixed point $(v(w), w)$ near $(0, w)$ for every $w$ near the origin, and $k$ is an admissible iteration of $\psi_{w}$. Furthermore, applying the inverse function theorem to $\psi_{w}^{k}$, we see that every $k$-periodic orbit of $\psi_{w}$ in a small neighborhood $U_{w}$ of $(v(w), w)$ in $V \times w$ is the fixed point $(v(w), w)$. Clearly, the size of $U_{w}$ is bounded from below when $w$ is close to the origin and $s$ is close to $s_{0}$. Thus, every $k$-periodic orbit of $\psi_{w}$ close to $0 \in V$ is in fact the fixed point of $\psi_{w}$. In particular, $v_{0}$ is the fixed point of $\psi_{w_{0}}$ and $z$ is a fixed point of $\varphi$. This concludes the proof of the proposition.

Remark 7.1. Combining the proof of Proposition 7.1 and the proof of the Shub-Sullivan theorem (see $[\mathbf{S S}]$ ), it is easy to see that for all admissible $k$ the index of $\varphi^{k}$ at $p$ is equal, up to a sign, to the index of $\varphi$ at $p$.

\section{References}

[AS1] A. Abbondandolo and M. Schwarz, On the Floer homology of cotangent bundles, Comm. Pure Appl. Math. 59 (2006), 254-316.

[AS2] A. Abbondandolo and M. Schwarz, Floer homology of cotangent bundles and the loop product, preprint, 2008.

[Ar] V.I. Arnold, Mathematical methods of classical mechanics, Springer-Verlag, New York, 1974.

[BC] J.F. Barraud, O. Cornea, Lagrangian intersections and the Serre spectral sequence, Ann. of Math. 166(3) (2007), 249-280.

[BPS] P. Biran, L. Polterovich and D. Salamon, Propagation in Hamiltonian dynamics and relative symplectic homology, Duke Math. J. 119 (2003), 65-118.

[Bo] F. Bourgeois, Introduction to contact homology, Lecture notes available at http://homepages.vub.ac.be/ fbourgeo/

[Ch] Y. Chekanov, Hofer's symplectic energy and Lagrangian intersections, in 'Contact and symplectic geometry (Cambridge, 1994)', 296-306; C.B. Thomas (ed.), Publ. Newton Inst., 8, Cambridge Univ. Press, Cambridge, 1996.

[CFHW] K. Cieliebak, A. Floer, H. Hofer and K. Wysocki, Applications of symplectic homology II: stability of the action spectrum, Math. Z. 223 (1996), 27-45.

[Co] C.C. Conley, Lecture at the University of Wisconsin, April 6, 1984.

[Fl1] A. Floer, Morse theory for Lagrangian intersections, J. Differential Geom. 28 (1988), 513-547.

[Fl2] A. Floer, The unregularized gradient flow of the symplectic action, Comm. Pure Appl. Math. 41 (1988), 775-813.

[Fl3] A. Floer, Cuplength estimates on Lagrangian intersections, Comm. Pure Appl. Math. 42 (1989), 335-356.

[Fl4] A. Floer, Witten's complex and infinite-dimensional Morse theory, J. Differential Geom. 30 (1989), 207-221. 
[Fl5] A. Floer, Symplectic fixed points and holomorphic spheres, Comm. Math. Phys. 120 (1989), 575-611.

[FHS] A. Floer, H. Hofer and D. Salamon, Transversality in elliptic Morse theory for the symplectic action, Duke Math. J. 80 (1995), 251-292.

[FrHa] J. Franks and M. Handel, Periodic points of Hamiltonian surface diffeomorphisms, Geom. Topol. 7 (2003), 713-756.

[FS] U. Frauenfelder and F. Schlenk, Hamiltonian dynamics on convex symplectic manifolds, Israel J. Math. 159 (2007) 1-56.

[Gi1] V.L. Ginzburg, Coisotropic intersections, Duke Math. J. 140 (2007), 111-163.

[Gi2] V.L. Ginzburg, The Conley conjecture, preprint, 2006, math.SG/0610956; to appear in Ann. of Math.

[GG1] V.L. Ginzburg and B.Z. Gürel, Periodic orbits of twisted geodesic flows and the Weinstein-Moser theorem, Comment. Math. Helv. 84 (2009), 865-907.

[GG2] V.L. Ginzburg and B.Z. Gürel, Action and index spectra and periodic orbits in Hamiltonian dynamics, Geom. Topol. 13 (2009), 2745-2805.

[GoHi] M. Goresky and N. Hingston, Loop products and closed geodesics, Duke Math. J. 150 (2009), 117-209.

[GrMe1] D. Gromoll and W. Meyer, On differentiable functions with isolated critical points, Topology 8 (1969), 361-369.

[GrMe2] D. Gromoll and W. Meyer, Periodic geodesics on compact Riemannian manifolds, J. Differential Geom. 3 (1969), 493-510.

[Gü] B.Z. Gürel, Totally non-coisotropic displacement and its applications to Hamiltonian dynamics, Comm. Contemp. Math. 10(6) (2008), 1103-1128.

[Hi] N. Hingston, Subharmonic solutions of Hamiltonian equations on tori, Ann. of Math. 170 (2009), 529-560.

[HS] H. Hofer and D. Salamon, Floer homology and Novikov rings, in 'The Floer memorial volume', 483-524, Progr. Math., 133, Birkhäuser, Basel, 1995.

[HZ] H. Hofer and E. Zehnder, Symplectic invariants and Hamiltonian dynamics, Birkäuser, Basel, 1994.

[Jo] J. Jost, Riemannian geometry and geometric analysis, Springer-Verlag, New York, 2002.

[LeC $]$ P. Le Calvez, Periodic orbits of Hamiltonian homeomorphisms of surfaces, Duke Math. J. 133 (2006), 125-184.

[Lo] Y. Long, Index theory for symplectic path with applications, Birkäuser, Basel, 2002.

[MS] D. McDuff and D. Salamon, J-holomorphic curves and symplectic topology, Colloquium publications, 52, AMS, Providence, RI, 2004.

[Po] M. Poźniak, Floer homology, Novikov rings and clean intersections, in 'Northern California symplectic geometry seminar', Amer. Math. Soc. Transl. Ser. 2, 196, 119-181, AMS, Providence, RI, 1999.

[PSS] S. Piunikhin, D. Salamon and M. Schwarz, Symplectic Floer-Donaldson theory and quantum cohomology, in 'Contact and symplectic geometry (Cambridge, 1994)', 171-200; C.B. Thomas (ed.), Publ. Newton Inst., 8, Cambridge Univ. Press, Cambridge, 1996. 
[Sa1] D.A. Salamon, Morse theory, the Conley index and Floer homology, Bull. L.M.S. 22 (1990), 113-140.

[Sa2] D.A. Salamon, Lectures on Floer homology, in 'Symplectic geometry and topology', (Y. Eliashberg and L. Traynor, eds.), IAS/Park City Mathematics series, 7, 1999, 143-230.

[SW] D.A. Salamon and J. Weber, Floer homology and the heat flow, Geom. Funct. Anal. 16 (2006), 1050-1138.

[SZ] D. Salamon and E. Zehnder, Morse theory for periodic solutions of Hamiltonian systems and the Maslov index, Comm. Pure Appl. Math. 45 (1992), 1303-1360.

[Sc1] M. Schwarz, Morse homology, Birkhäuser, Basel, 1993.

[Sc2] M. Schwarz, Cohomology operations from $S^{1}$-cobordisms in Floer theory, Ph.D. thesis, Dissertation ETH 11182, Swiss Federal Institute of Technology, Zurich, 1995.

[Sc3] M. Schwarz, On the action spectrum for closed symplectically aspherical manifolds, Pacific J. Math. 193 (2000), 419-461.

[SS] M. Shub and D. Sullivan, A remark on the Lefschetz fixed point formula for differentiable maps, Topology 13 (1974), 189-191.

[Vi1] C. Viterbo, Symplectic topology as the geometry of generating functions, Math. Ann. 292 (1992), 685-710.

[Vi2] C. Viterbo, Functors and computations in Floer cohomology, I, Geom. Funct. Anal. 9 (1999), 985-1033.

[We1] A. Weinstein, Symplectic manifolds and their Lagrangian submanifolds, Adv. Math. 6 (1971), 329-346.

[We2] A. Weinstein, Lectures on symplectic manifolds, Expository lectures from the CBMS Regional Conference held at the University of North Carolina, March 812, 1976. Regional Conference Series in Mathematics, No. 29, AMS, Providence, RI, 1977.

[Yo] J.A. Yorke, Periods of periodic solutions and the Lipschitz constant, Proc. Amer. Math. Soc. 22 (1969), 509-512.

Department of Mathematics, UC Santa Cruz, Santa Cruz, CA 95064

E-mail address: ginzburg@math.ucsc.edu

Department of Mathematics, VAnderbilt University, Nashville, TN 37240

E-mail address: basak.gurel@vanderbilt.edu

Received 6/15/2009, accepted 1/19/2010

The work is partially supported by the NSF and by the faculty research funds of the University of California, Santa Cruz.

The authors are grateful to the referee for useful remarks. 
